Supplement of Geosci. Model Dev., 8, 2399-2417, 2015

http://www.geosci-model-dev.net/8/2399/2015/

doi:10.5194/gmd-8-2399-2015-supplement

(C) Author(s) 2015. CC Attribution 3.0 License.

(c) (i)

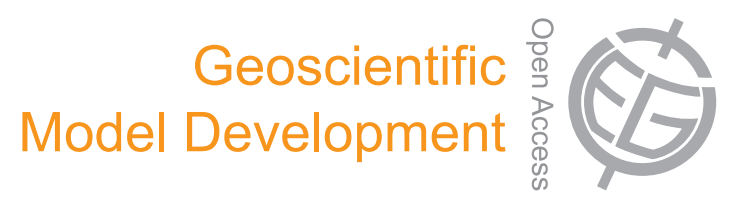

Supplement of

\title{
The Yale Interactive terrestrial Biosphere model version 1.0: description, evaluation and implementation into NASA GISS ModelE2
}

X. Yue and N. Unger

Correspondence to: X. Yue (xuyueseas@gmail.com)

The copyright of individual parts of the supplement might differ from the CC-BY 3.0 licence. 


\section{Supporting information}

\section{Soil carbon pool spin-up process using YIBs-offline}

Studies investigating terrestrial carbon fluxes usually initialize models for the preindustrial period when human perturbations are negligible and soil carbon pools are considered to be at an equilibrium state (Huntzinger et al., 2013; Sitch et al., 2015). YIBs requires (minimum) hourly meteorological input data fields, which are only available from 1980. Therefore, to spin-up YIBs, we adapt the approach of Wutzler and Reichstein (2007), who applied a transient correction to the soil carbon pools so that the simulated stocks match observations.

We apply YIBs-offline to spin-up the soil carbon pools that provide the initial conditions for all 3 experimental configurations (site-level, YIBs-offline and YIBs-online). The spin-up process proceeds in 2 stages. In the first stage, we set a uniform initial height $H_{0}$ for each PFT (Table 1) and run YIBs-offline for 60 years using fixed $\mathrm{CO}_{2}$ concentrations and meteorological forcings for the year 1980 to allow vegetation carbon to equilibrate. By the end of the first stage spin-up run, the year-to-year variations of global average tree height, LAI, GPP, and NPP are all within $\pm 0.05 \%$. In the second stage, the derived equilibrium tree heights from the first stage and the global soil carbon content at the top 30 centimeters (Batjes, 2009) provide the initial conditions for an 80-year run (again using fixed $\mathrm{CO}_{2}$ concentrations and meteorological forcings for the year 1980) until the transient NEE is equal to $-2 \mathrm{Pg} \mathrm{C} \mathrm{a}^{-1}$, a value supported by observations and multi-model ensembles (Piao et al., 2013).

Following this 2 stage $60+80=140$ year total spin-up, the derived soil carbon pools represent the state for the year 1980. For YIBs site level simulations: the derived spun-up soil carbon pools for 1980 are used as initial conditions. For YIBs-offline simulations: the derived spun-up soil carbon pools and equilibrium tree heights for 1980 are used as initial conditions for the 1980-2011 transient period. For the on-line simulations with NASA ModelE2-YIBs: the entire 140-year process described above is repeated with fixed WFDEI meteorology and atmospheric $\mathrm{CO}_{2}$ conditions for the year 2000 values. The 
derived spun-up soil carbon pools and equilibrium tree heights for 2000 are used as initial conditions for the present-day climatological coupled global carbon-chemistry-climate simulation. 
(a) Shrub $f_{D}$-GPP correlations

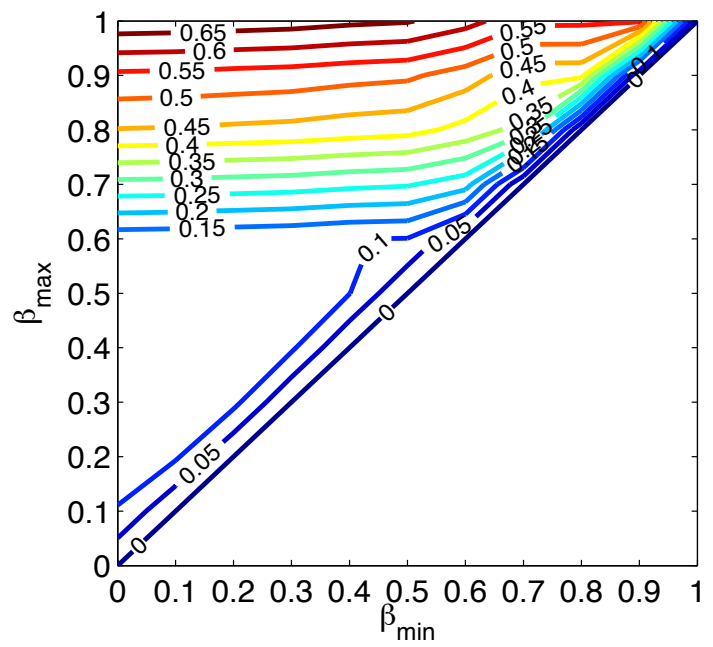

(b) Grass $f_{D}$-GPP correlations

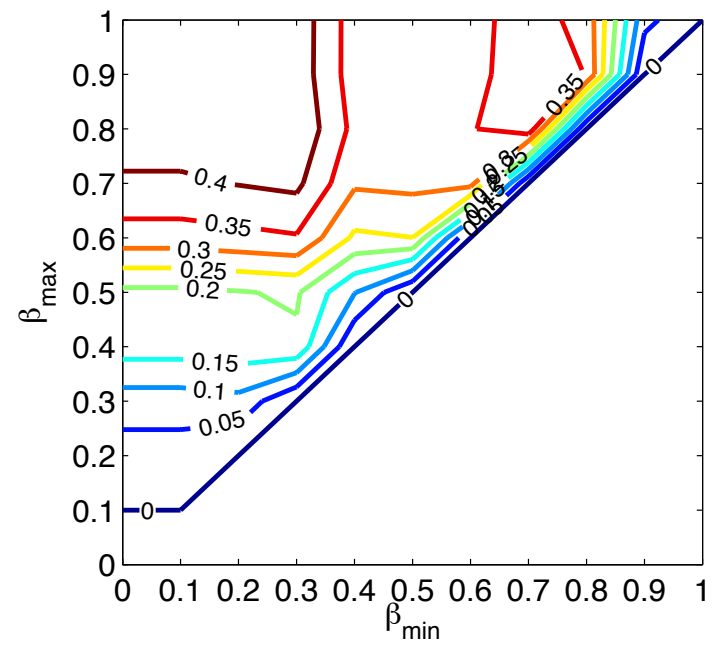

Figure S1. Average correlation coefficients between monthly GPP and droughtdependent phenology $f_{D}$ at (a) shrub and (b) grass sites derived with different water stress thresholds $\left(\beta_{\min }\right.$ and $\left.\beta_{\max }\right)$. The results are based on 14 warm sites, 8 for shrub and 6 for grass, with annual soil temperature $>12^{\circ} \mathrm{C}$ as shown in Fig. 2. 
$\operatorname{BE}-\operatorname{Bra}\left(4.5^{\circ} \mathrm{E}, 51.3^{\circ} \mathrm{N}\right)$

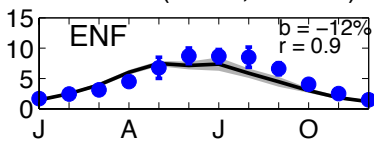

$\mathrm{CA}-\mathrm{Ca} 2\left(125.3^{\circ} \mathrm{W}, 49.9^{\circ} \mathrm{N}\right)$

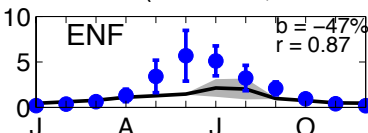

CA-NS1 $\left(98.5^{\circ} \mathrm{W}, 55.9^{\circ} \mathrm{N}\right)$

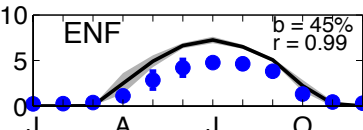

CA-NS5 $\left(98.5^{\circ} \mathrm{W}, 55.9^{\circ} \mathrm{N}\right)$

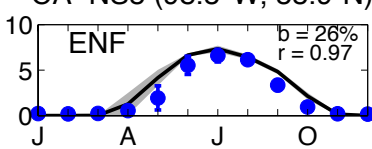

CA-Qfo $\left(74.3^{\circ} \mathrm{W}, 49.7^{\circ} \mathrm{N}\right)$

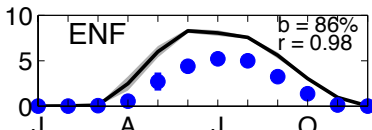

CA-TP4 $\left(80.4^{\circ} \mathrm{W}, 42.7^{\circ} \mathrm{N}\right)$

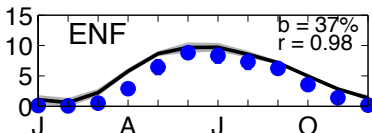

DE-Wet $\left(11.5^{\circ} \mathrm{E}, 50.5^{\circ} \mathrm{N}\right)$

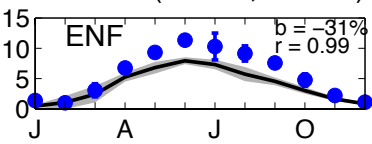

FR-LBr $\left(0.8^{\circ} \mathrm{W}, 44.7^{\circ} \mathrm{N}\right)$

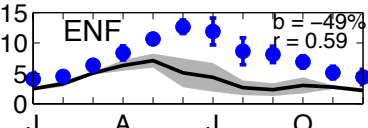

IT-SRo $\left(10.3^{\circ} \mathrm{E}, 43.7^{\circ} \mathrm{N}\right)$

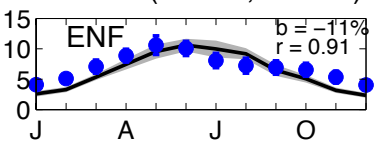

SE-Nor $\left(17.5^{\circ} \mathrm{E}, 60.1^{\circ} \mathrm{N}\right)$

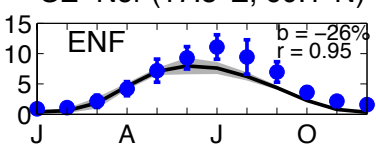

BE-Jal $\left(6.1^{\circ} \mathrm{E}, 50.6^{\circ} \mathrm{N}\right)$

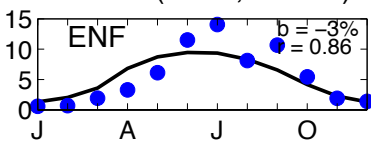

$\mathrm{CA}-\mathrm{Ca} 3\left(124.9^{\circ} \mathrm{W}, 49.5^{\circ} \mathrm{N}\right)$

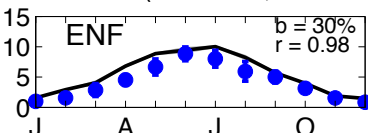

CA-NS2 $\left(98.5^{\circ} \mathrm{W}, 55.9^{\circ} \mathrm{N}\right)$

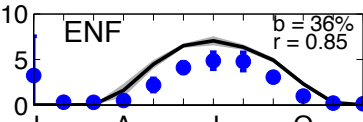

CA-Obs $\left(105.1^{\circ} \mathrm{W}, 54^{\circ} \mathrm{N}\right)$

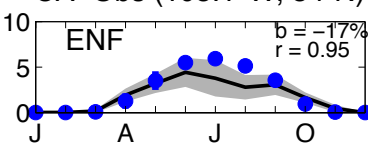

CA-SJ1 $\left(104.7^{\circ} \mathrm{W}, 53.9^{\circ} \mathrm{N}\right)$

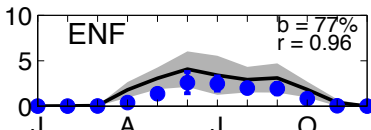

CZ-BK1 $\left(18.5^{\circ} \mathrm{E}, 49.5^{\circ} \mathrm{N}\right)$

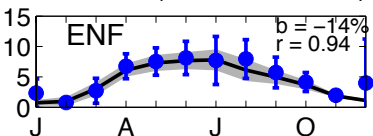

ES-ES1 $\left(0.3^{0} \mathrm{~W}, 39.3^{\circ} \mathrm{N}\right)$

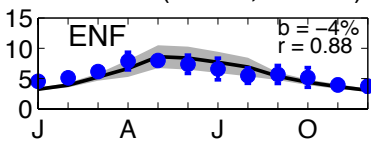

IL-Yat $\left(35.1^{\circ} \mathrm{E}, 31.3^{\circ} \mathrm{N}\right)$

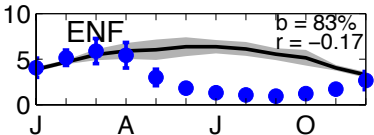

$\mathrm{NL}-$ Loo $\left(5.7^{\circ} \mathrm{E}, 52.2^{\circ} \mathrm{N}\right)$

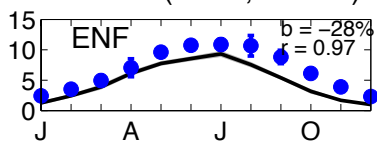

SE-Sk1 $\left(17.9^{\circ} \mathrm{E}, 60.1^{\circ} \mathrm{N}\right)$

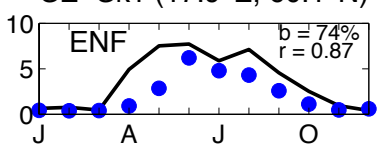

BE-Vie $\left(6^{\circ} \mathrm{E}, 50.3^{\circ} \mathrm{N}\right)$

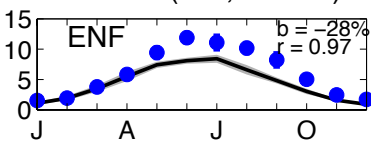

CA-Gro $\left(82.2^{\circ} \mathrm{W}, 48.2^{\circ} \mathrm{N}\right)$

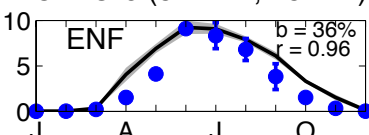

CA-NS3 $\left(98.4^{\circ} \mathrm{W}, 55.9^{\circ} \mathrm{N}\right)$
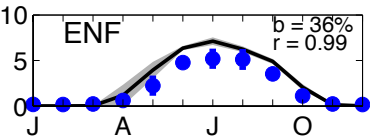

CA-Ojp $\left(104.7^{\circ} \mathrm{W}, 53.9^{\circ} \mathrm{N}\right)$

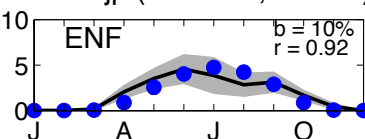

CA-SJ2 $\left(104.6^{\circ} \mathrm{W}, 53.9^{\circ} \mathrm{N}\right)$

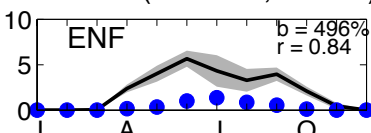

DE-Bay $\left(11.9^{\circ} \mathrm{E}, 50.1^{\circ} \mathrm{N}\right)$

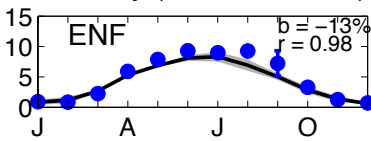

$\mathrm{Fl}-\mathrm{Hyy}\left(24.3^{\circ} \mathrm{E}, 61.8^{\circ} \mathrm{N}\right)$

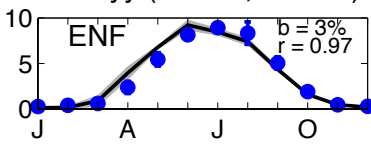

IT-Lav $\left(11.3^{\circ} \mathrm{E}, 46^{\circ} \mathrm{N}\right)$

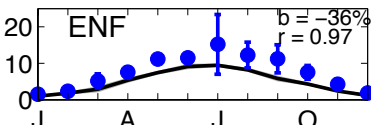

RU-Fyo $\left(32.9^{\circ} \mathrm{E}, 56.5^{\circ} \mathrm{N}\right)$

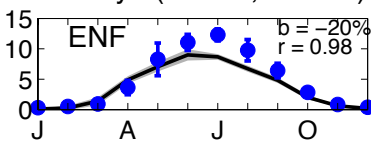

SE-Sk2 $\left(17.8^{\circ} \mathrm{E}, 60.1^{\circ} \mathrm{N}\right)$

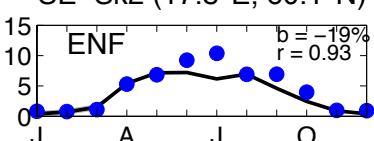

$\mathrm{CA}-\mathrm{Ca} 1\left(125.3^{\circ} \mathrm{W}, 49.9^{\circ} \mathrm{N}\right)$

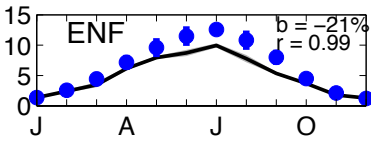

CA-Man $\left(98.5^{\circ} \mathrm{W}, 55.9^{\circ} \mathrm{N}\right)$

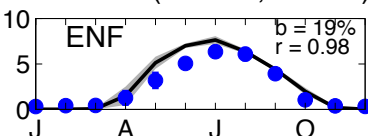

CA-NS4 $\left(98.4^{\circ} \mathrm{W}, 55.9^{\circ} \mathrm{N}\right)$
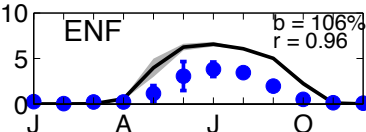

CA-Qcu $\left(74^{\circ} \mathrm{W}, 49.3^{\circ} \mathrm{N}\right)$

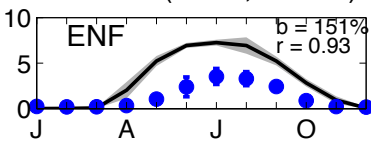

CA-SJ3 $\left(104.6^{\circ} \mathrm{W}, 53.9^{\circ} \mathrm{N}\right)$
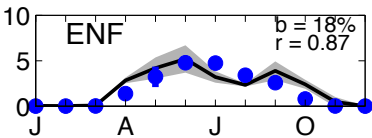

DE-Tha $\left(13.6^{\circ} \mathrm{E}, 51^{\circ} \mathrm{N}\right)$

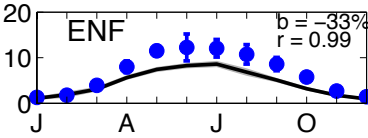

$\mathrm{FI}-\mathrm{Sod}\left(26.6^{\circ} \mathrm{E}, 67.4^{\circ} \mathrm{N}\right)$

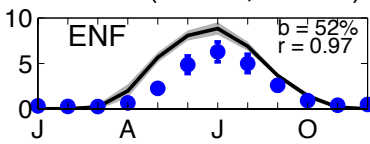

IT-Ren $\left(11.4^{\circ} \mathrm{E}, 46.6^{\circ} \mathrm{N}\right)$

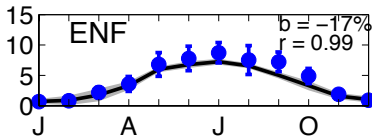

SE-Fla $\left(19.5^{\circ} \mathrm{E}, 64.1^{\circ} \mathrm{N}\right)$

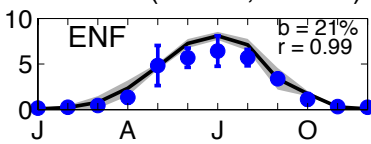

UK-Gri $\left(3.8^{\circ} \mathrm{W}, 56.6^{\circ} \mathrm{N}\right)$

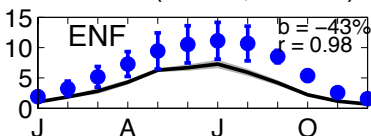

Figure S2. Comparison of simulated (solid lines) and observed (blue points) monthly gross primary productivity (GPP) at 145 sites from FLUXNET and NACP network. Both simulations and observations are averaged over the period when measurements are available (Table S1). Error bars represent one standard deviation of observations while shadings represent that of simulations. The relative bias and correlation coefficient are shown on each panel. The land types include evergreen needleleaf forest (ENF), evergreen broadleaf forest (EBF), deciduous broadleaf forest (DBF), shrublands (SHR), grasslands (GRA), and croplands (CRO). Units of GPP: $\mathrm{g} \mathrm{C} \mathrm{m}^{-2}$ day $^{-1}$. 


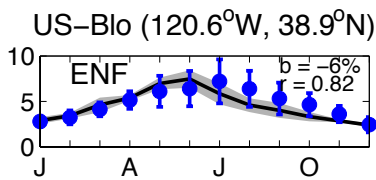

US-Me2 $\left(121.6^{\circ} \mathrm{W}, 44.5^{\circ} \mathrm{N}\right)$

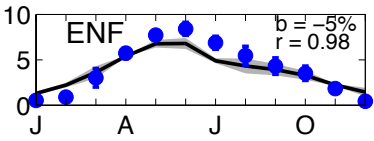

US-NR1 $\left(105.5^{\circ} \mathrm{W}, 40^{\circ} \mathrm{N}\right)$

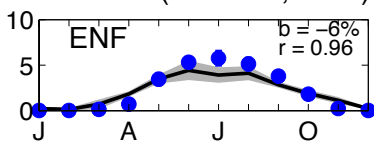

US-SP3 $\left(82.2^{\circ} \mathrm{W}, 29.8^{\circ} \mathrm{N}\right)$

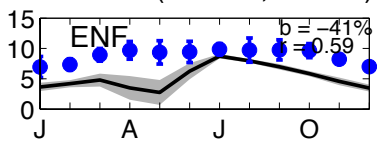

BR-Sa3 $\left(55^{\circ} \mathrm{W}, 3^{\circ} \mathrm{S}\right)$

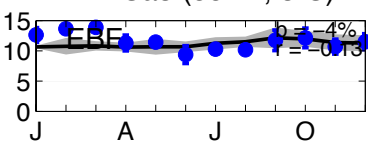

IT-Lec $\left(11.3^{\circ} \mathrm{E}, 43.3^{\circ} \mathrm{N}\right)$

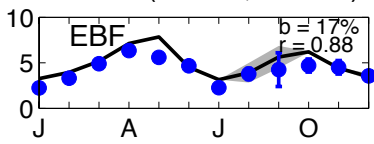

DE-Hai $\left(10.5^{\circ} \mathrm{E}, 51.1^{\circ} \mathrm{N}\right)$

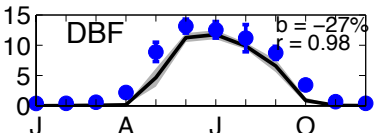

IT-Col $\left(13.6^{\circ} \mathrm{E}, 41.8^{\circ} \mathrm{N}\right)$

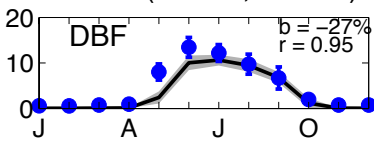

IT-Ro2 $\left(11.9^{\circ} \mathrm{E}, 42.4^{\circ} \mathrm{N}\right)$

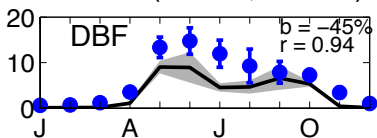

US-Ha1 $\left(72.2^{\circ} \mathrm{W}, 42.5^{\circ} \mathrm{N}\right)$

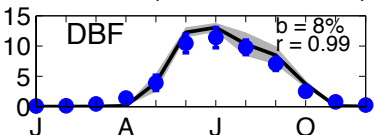

US-Dk3 $\left(79.1^{\circ} \mathrm{W}, 36^{\circ} \mathrm{N}\right)$

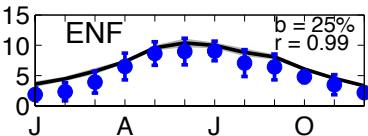

US-Me3 $\left(121.6^{\circ} \mathrm{W}, 44.3^{\circ} \mathrm{N}\right)$

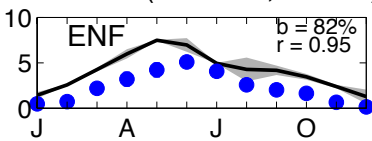

US-PFa $\left(90.3^{\circ} \mathrm{W}, 45.9^{\circ} \mathrm{N}\right)$

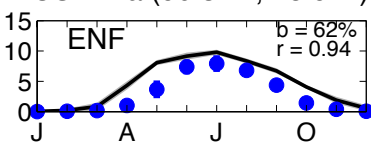

US-Syv $\left(89.3^{\circ} \mathrm{W}, 46.2^{\circ} \mathrm{N}\right)$

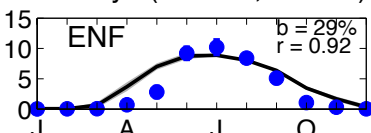

FR-Pue $\left(3.6^{\circ} \mathrm{E}, 43.7^{\circ} \mathrm{N}\right)$

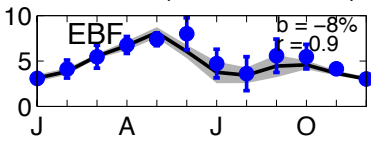

PT-Esp $\left(8.6^{\circ} \mathrm{W}, 38.6^{\circ} \mathrm{N}\right)$

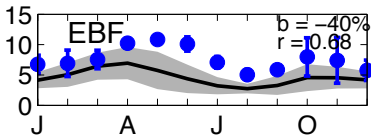

DK-Sor $\left(11.6^{\circ} \mathrm{E}, 55.5^{\circ} \mathrm{N}\right)$

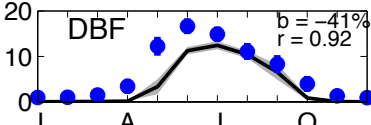

IT-Non $\left(11.1^{\circ} \mathrm{E}, 44.7^{\circ} \mathrm{N}\right)$

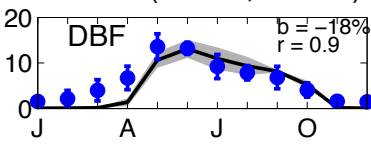

UK-Ham $\left(0.9^{\circ} \mathrm{W}, 51.2^{\circ} \mathrm{N}\right)$

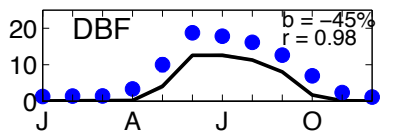

US-MMS $\left(86.4^{\circ} \mathrm{W}, 39.3^{\circ} \mathrm{N}\right)$

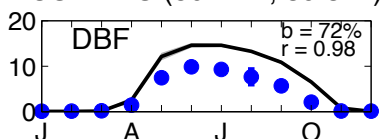

US-Ho1 $\left(68.7^{\circ} \mathrm{W}, 45.2^{\circ} \mathrm{N}\right)$

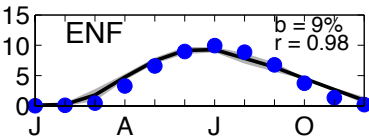

US-Me4 $\left(121.6^{\circ} \mathrm{W}, 44.5^{\circ} \mathrm{N}\right)$

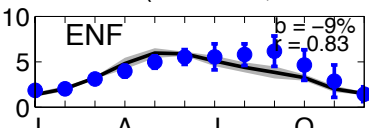

US-SP1 $\left(82.2^{\circ} \mathrm{W}, 29.7^{\circ} \mathrm{N}\right)$

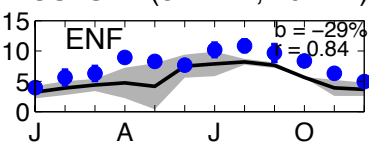

AU-Tum $\left(148.2^{\circ} \mathrm{E}, 35.7^{\circ} \mathrm{S}\right)$

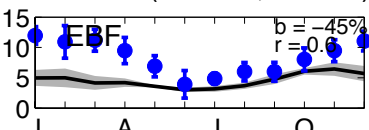

ID-Pag $\left(114^{\circ} \mathrm{E}, 2.3^{\circ} \mathrm{N}\right)$

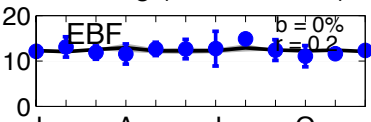

PT-Mi1 $\left(8^{\circ} \mathrm{W}, 38.5^{\circ} \mathrm{N}\right)$

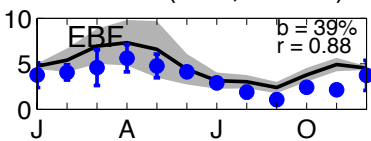

FR-Fon $\left(2.8^{\circ} \mathrm{E}, 48.5^{\circ} \mathrm{N}\right)$

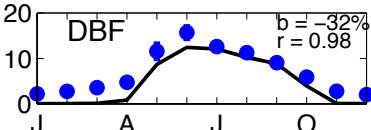

IT-PT1 $\left(9.1^{\circ} \mathrm{E}, 45.2^{\circ} \mathrm{N}\right)$

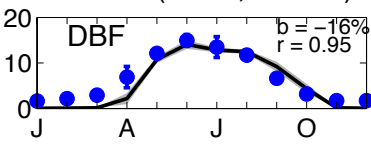

UK-PL3 $\left(1.3^{\circ} \mathrm{W}, 51.5^{\circ} \mathrm{N}\right)$

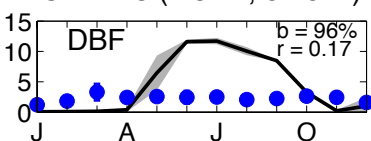

US-MOz $\left(92.2^{\circ} \mathrm{W}, 38.7^{\circ} \mathrm{N}\right)$

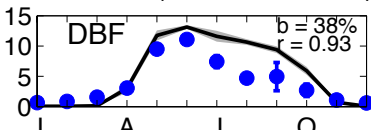

US-Ho2 $\left(68.7^{\circ} \mathrm{W}, 45.2^{\circ} \mathrm{N}\right)$

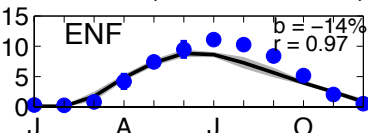

US-Me5 $\left(121.6^{\circ} \mathrm{W}, 44.4^{\circ} \mathrm{N}\right)$

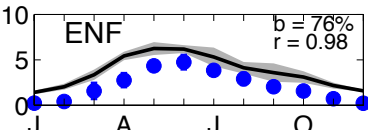

US-SP2 $\left(82.2^{\circ} \mathrm{W}, 29.8^{\circ} \mathrm{N}\right)$

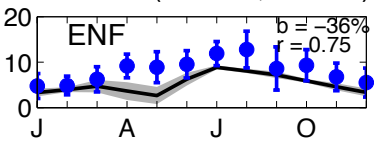

AU-Wac $\left(145.2^{\circ} \mathrm{E}, 37.4^{\circ} \mathrm{S}\right)$

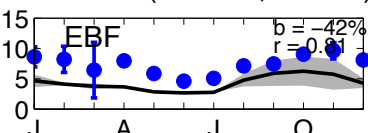

IT-Cpz $\left(12.4^{\circ} \mathrm{E}, 41.7^{\circ} \mathrm{N}\right)$

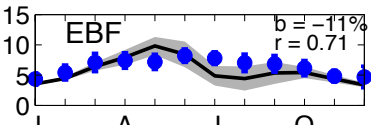

CA-Oas $\left(106.2^{\circ} \mathrm{W}, 53.6^{\circ} \mathrm{N}\right)$

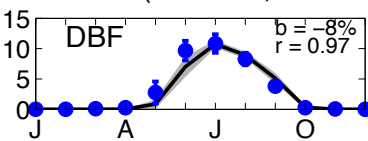

FR-Hes $\left(7.1^{\circ} \mathrm{E}, 48.7^{\circ} \mathrm{N}\right)$

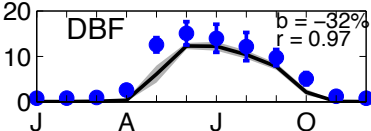

IT-Ro1 $\left(11.9^{\circ} \mathrm{E}, 42.4^{\circ} \mathrm{N}\right)$

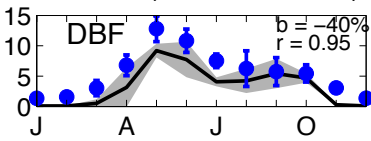

US-Bar $\left(71.3^{\circ} \mathrm{W}, 44.1^{\circ} \mathrm{N}\right)$

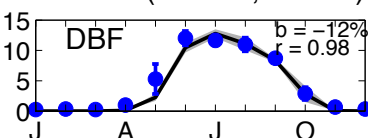

US-Oho $\left(83.8^{\circ} \mathrm{W}, 41.6^{\circ} \mathrm{N}\right)$

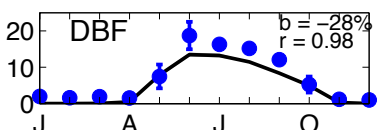

Figure S2. Continued. 


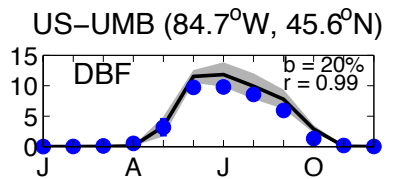

AU-How $\left(131.2^{\circ} \mathrm{E}, 12.5^{\circ} \mathrm{S}\right)$

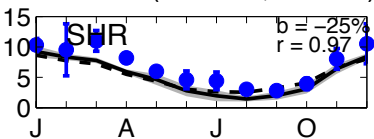

CA-NS7 $\left(99.9^{\circ} \mathrm{W}, 56.6^{\circ} \mathrm{N}\right)$

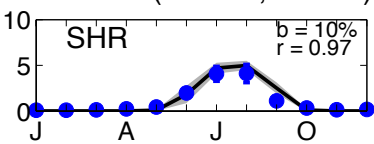

IT-Pia $\left(10.1^{\circ} \mathrm{E}, 42.6^{\circ} \mathrm{N}\right)$

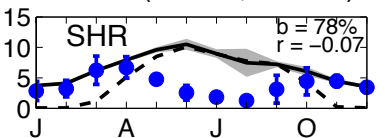

UK-AMo $\left(3.2^{\circ} \mathrm{W}, 55.8^{\circ} \mathrm{N}\right)$

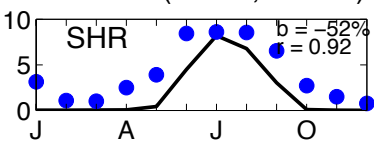

ZA-Kru $\left(31.5^{\circ} \mathrm{E}, 25^{\circ} \mathrm{S}\right)$

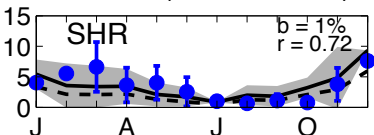

DE-Gri $\left(13.5^{\circ} \mathrm{E}, 51^{\circ} \mathrm{N}\right)$

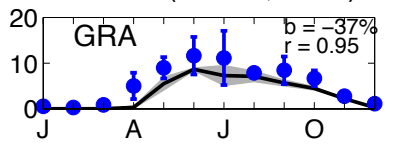

FR-Lq1 $\left(2.7^{\circ} \mathrm{E}, 45.6^{\circ} \mathrm{N}\right)$

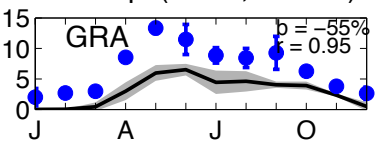

IE-Dri $\left(8.8^{\circ} \mathrm{W}, 52^{\circ} \mathrm{N}\right)$

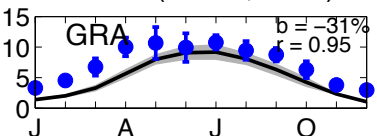

IT-Mal $\left(11.7^{\circ} \mathrm{E}, 46.1^{\circ} \mathrm{N}\right)$

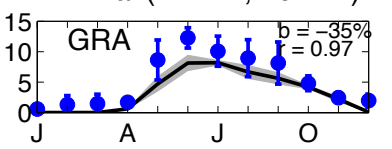

US-WBW $\left(84.3^{\circ} \mathrm{W}, 36^{\circ} \mathrm{N}\right)$

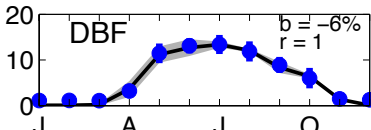

BW-Ma1 $\left(23.6^{\circ} \mathrm{E}, 19.9^{\circ} \mathrm{S}\right)$

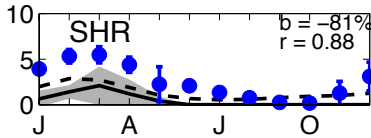

CA-WP1 $\left(112.5^{\circ} \mathrm{W}, 55^{\circ} \mathrm{N}\right)$

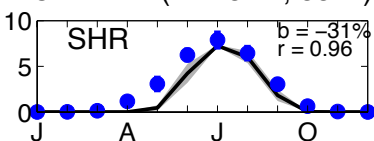

PL-wet $\left(16.3^{\circ} \mathrm{E}, 52.8^{\circ} \mathrm{N}\right)$

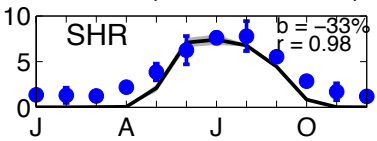

US-Los $\left(90^{\circ} \mathrm{W}, 46.1^{\circ} \mathrm{N}\right)$

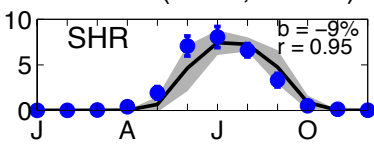

AT-Neu $\left(11.3^{\circ} \mathrm{E}, 47.1^{\circ} \mathrm{N}\right)$

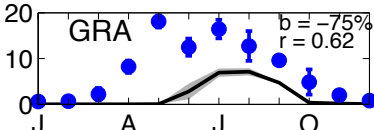

DE-Meh $\left(10.7^{\circ} \mathrm{E}, 51.3^{\circ} \mathrm{N}\right)$

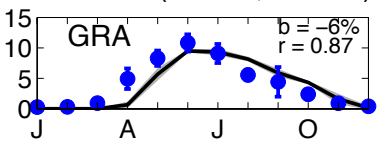

FR-Lq2 $\left(2.7^{\circ} \mathrm{E}, 45.6^{\circ} \mathrm{N}\right)$

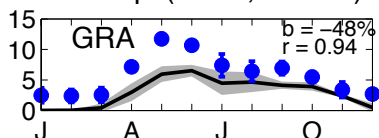

IT-Amp $\left(13.6^{\circ} \mathrm{E}, 41.9^{\circ} \mathrm{N}\right)$

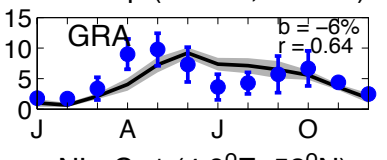

$\mathrm{NL}-\mathrm{Ca} 1\left(4.9^{\circ} \mathrm{E}, 52^{\circ} \mathrm{N}\right)$

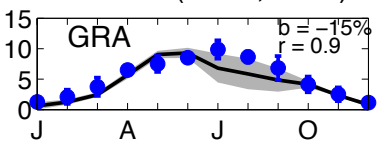

US-WCr $\left(90.1^{\circ} \mathrm{W}, 45.8^{\circ} \mathrm{N}\right)$

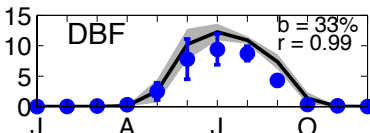

CA-Mer $\left(75.5^{\circ} \mathrm{W}, 45.4^{\circ} \mathrm{N}\right)$

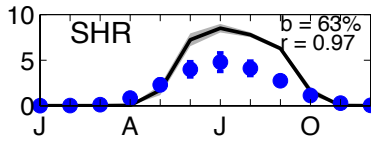

ES-LMa $\left(5.8^{\circ} \mathrm{W}, 39.9^{\circ} \mathrm{N}\right)$

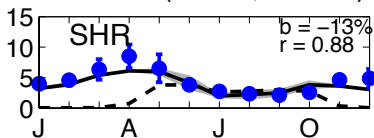

SE-Deg $\left(19.6^{\circ} \mathrm{E}, 64.2^{\circ} \mathrm{N}\right)$

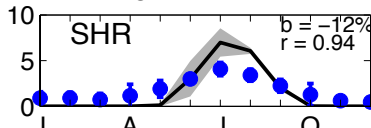

US-SO2 $\left(116.6^{\circ} \mathrm{W}, 33.4^{\circ} \mathrm{N}\right)$

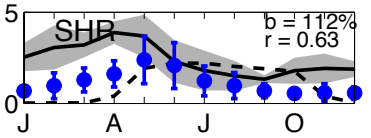

CA-Let $\left(112.9^{\circ} \mathrm{W}, 49.7^{\circ} \mathrm{N}\right)$
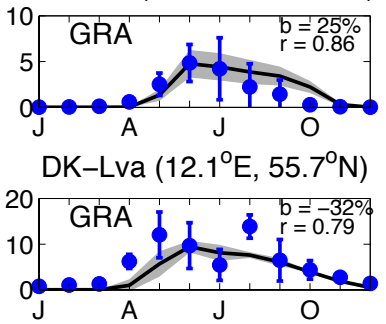

HU-Bug $\left(19.6^{\circ} \mathrm{E}, 46.7^{\circ} \mathrm{N}\right)$

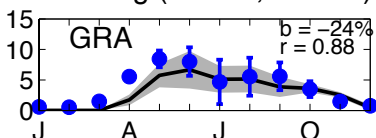

IT-LMa $\left(7.2^{\circ} \mathrm{E}, 45.6^{\circ} \mathrm{N}\right)$

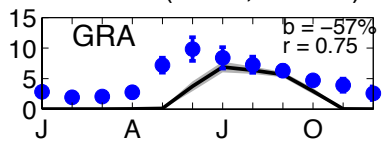

$\mathrm{NL}-\operatorname{Hor}\left(5.1^{\circ} \mathrm{E}, 52^{\circ} \mathrm{N}\right)$

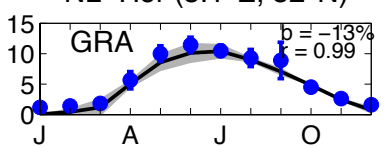

AU-Fog $\left(131.3^{\circ} \mathrm{E}, 12.5^{\circ} \mathrm{S}\right)$

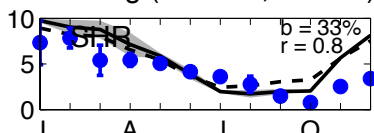

CA-NS6 $\left(99^{\circ} \mathrm{W}, 55.9^{\circ} \mathrm{N}\right)$

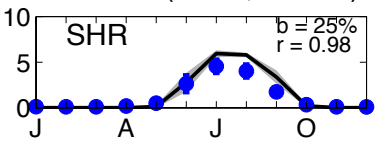

$\mathrm{FI}-\mathrm{Kaa}\left(27.3^{\circ} \mathrm{E}, 69.1^{\circ} \mathrm{N}\right)$

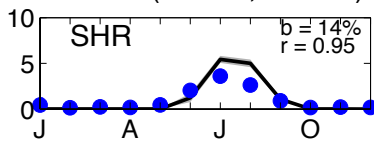

SE-Faj $\left(13.6^{\circ} \mathrm{E}, 56.3^{\circ} \mathrm{N}\right)$

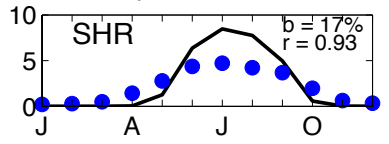

US-Ton $\left(121^{\circ} \mathrm{W}, 38.4^{\circ} \mathrm{N}\right)$

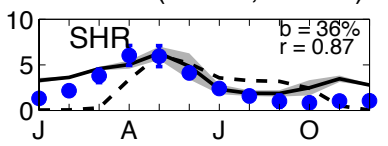

$\mathrm{CH}-\mathrm{Oe1}\left(7.7^{\circ} \mathrm{E}, 47.3^{\circ} \mathrm{N}\right)$

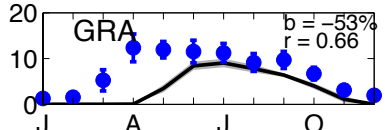

ES-VDA $\left(1.4^{\circ} \mathrm{E}, 42.2^{\circ} \mathrm{N}\right)$

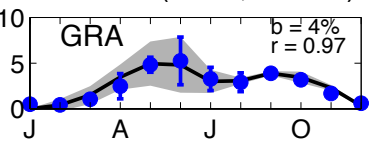

HU-Mat $\left(19.7^{\circ} \mathrm{E}, 47.8^{\circ} \mathrm{N}\right)$

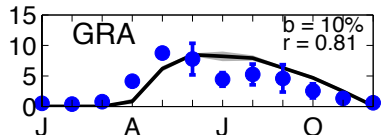

IT-MBo $\left(11^{\circ} \mathrm{E}, 46^{\circ} \mathrm{N}\right)$

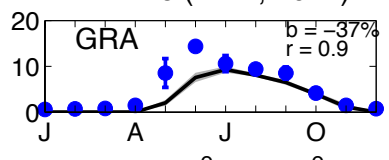

PT-Mi2 $\left(8^{\circ} \mathrm{W}, 38.5^{\circ} \mathrm{N}\right)$

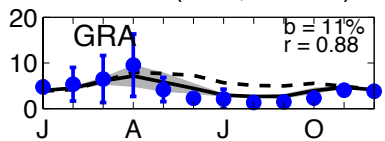

Figure S2. Continued. Dashed lines in some shrub and grass sites represent simulations with cold phenology alone. 

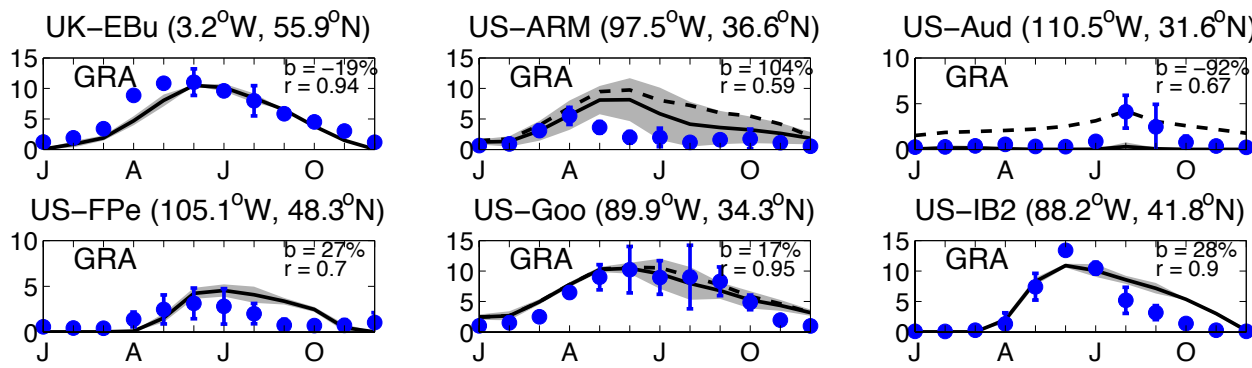

US-Bkg $\left(96.8^{\circ} \mathrm{W}, 44.3^{\circ} \mathrm{N}\right)$

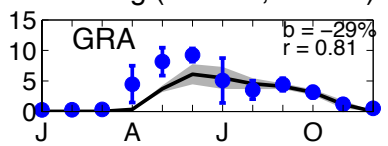

US-IB2 $\left(88.2^{\circ} \mathrm{W}, 41.8^{\circ} \mathrm{N}\right)$
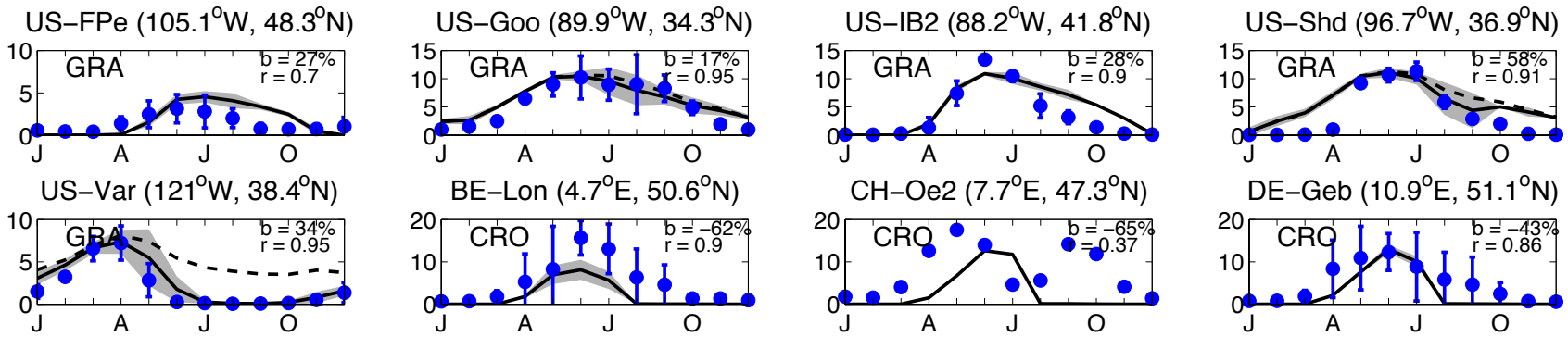

$\mathrm{CH}-\mathrm{Oe} 2\left(7.7^{\circ} \mathrm{E}, 47.3^{\circ} \mathrm{N}\right)$

DE-Geb $\left(10.9^{\circ} \mathrm{E}, 51.1^{\circ} \mathrm{N}\right)$
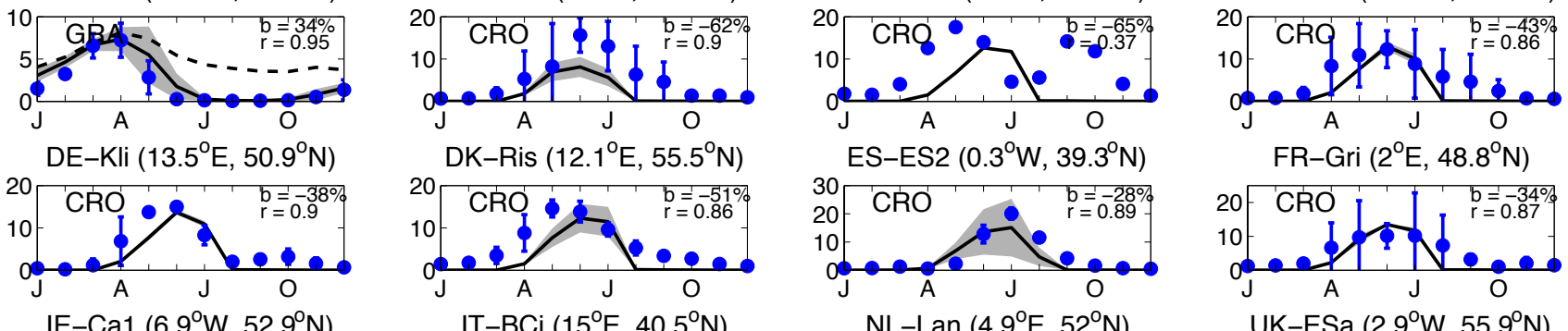

FR-Gri $\left(2^{\circ} \mathrm{E}, 48.8^{\circ} \mathrm{N}\right)$
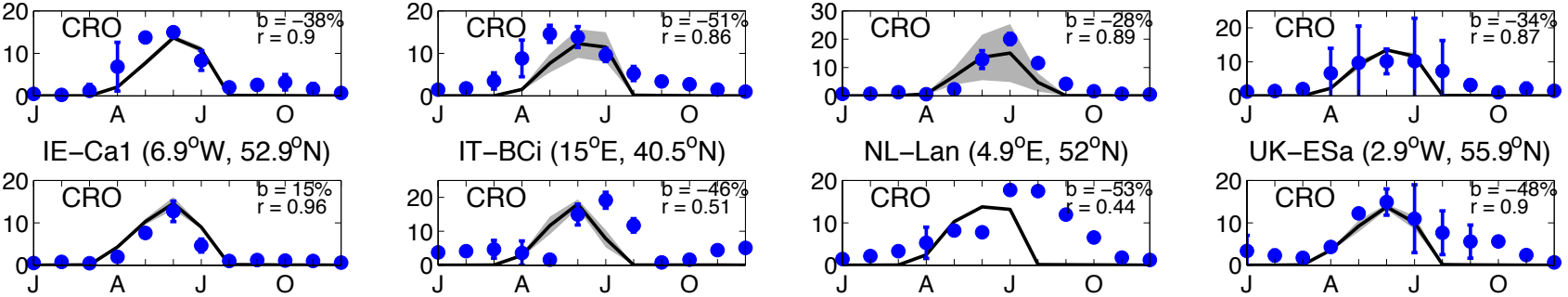

UK-ESa $\left(2.9^{\circ} \mathrm{W}, 55.9^{\circ} \mathrm{N}\right)$

US-Bo1 $\left(88.3^{\circ} \mathrm{W}, 40^{\circ} \mathrm{N}\right)$

US-IB1 $\left(88.2^{\circ} \mathrm{W}, 41.9^{\circ} \mathrm{N}\right)$

US-Ne1 $\left(96.5^{\circ} \mathrm{W}, 41.2^{\circ} \mathrm{N}\right)$
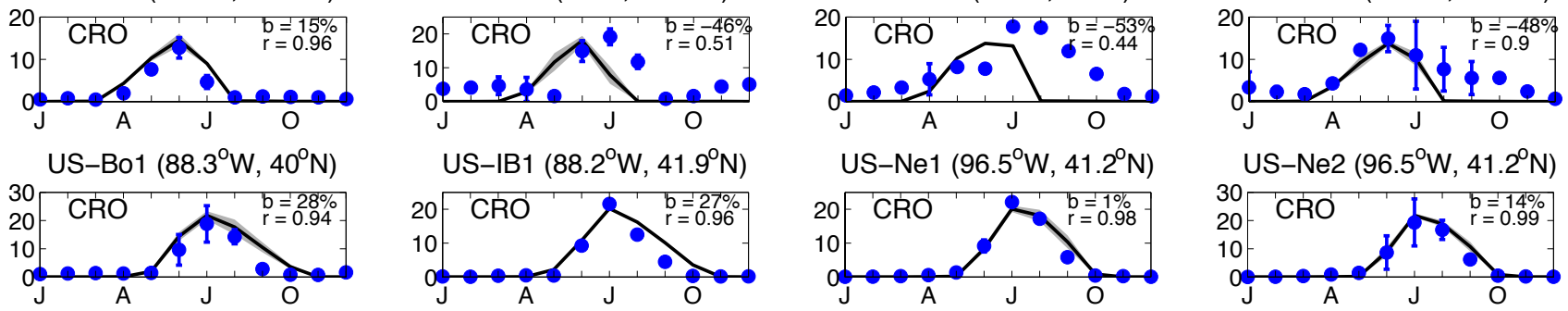

US-Ne2 $\left(96.5^{\circ} \mathrm{W}, 41.2^{\circ} \mathrm{N}\right)$
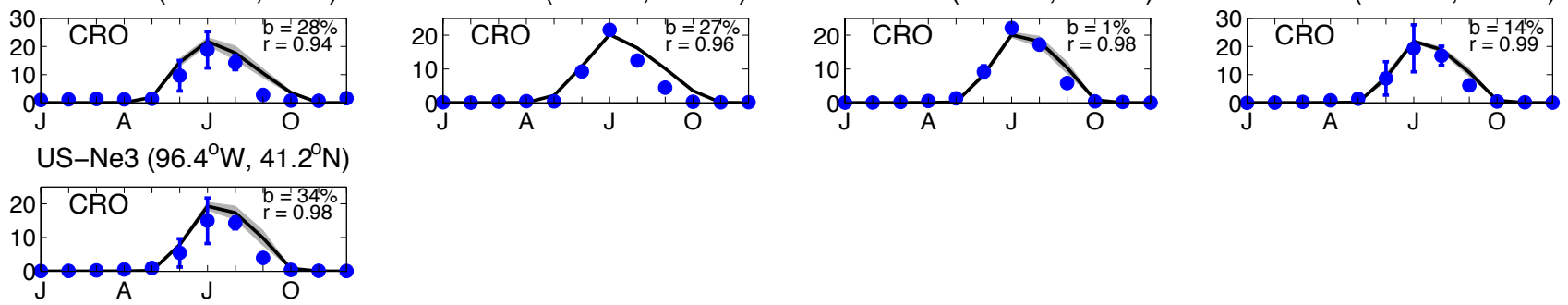

Figure S2. Continued. Dashed lines in some grass sites represent simulations with cold phenology alone. 
(a) Histogram of $\Delta R$ with drought phenology

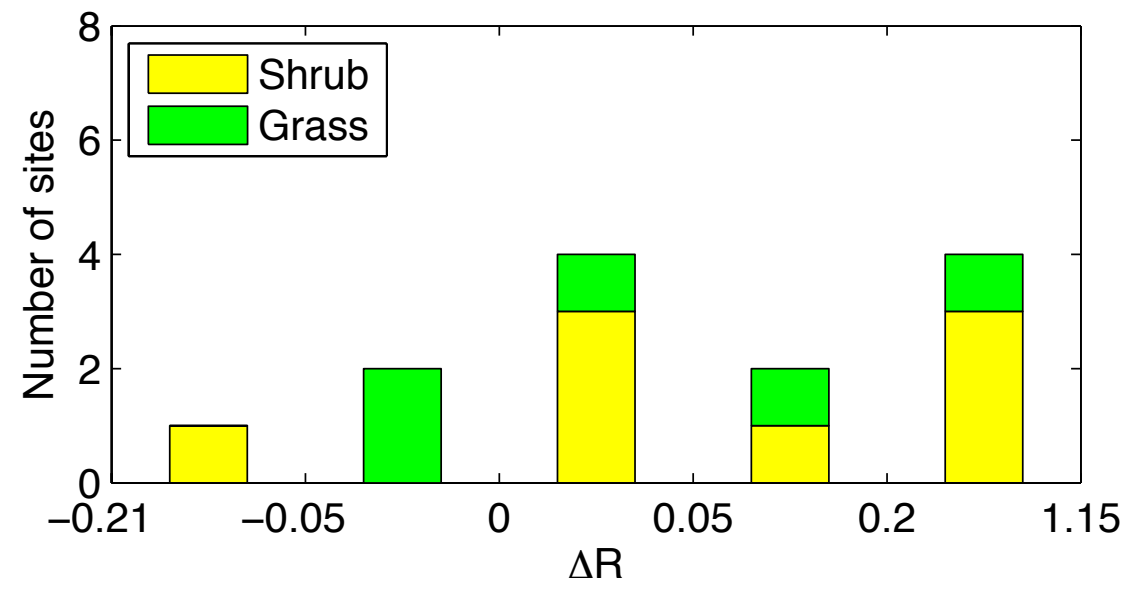

(b) Histogram of $\triangle \mathrm{RMSE}$ with drought phenology

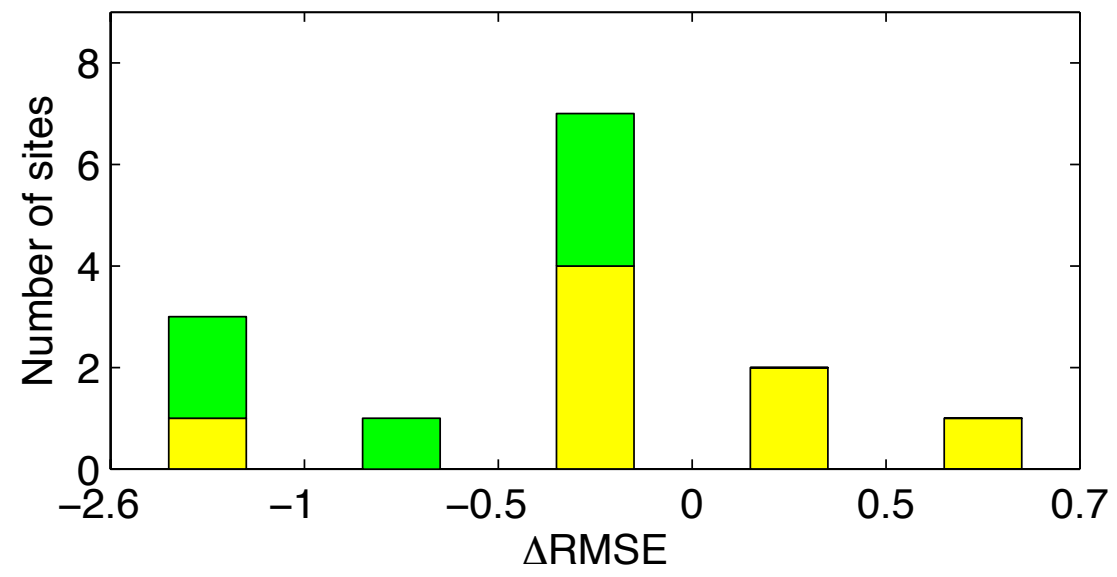

Figure S3. Histogram of changes in (a) correlation coefficients $(R)$ and (b) root mean square error (RMSE) between the observed and simulated GPP after the inclusion of drought phenology at 14 warm sites (annual soil temperature $>12{ }^{\circ} \mathrm{C}$ ). Each bar represents the number of sites where $\Delta R$ or $\triangle$ RMSE of simulations fall between the specific ranges as defined by the $\mathrm{x}$-axis intervals. The minimum and maximum of $\Delta R$ or $\triangle$ RMSE are indicated as the two ends of $x$-axis in the plots. The distribution of $x$-axis is asymmetric. Detailed comparisons at these sites are presented in Fig. S2. Units of RMSE: $\mathrm{g} \mathrm{C} \mathrm{m}^{-2}$ day $^{-1}$. 
BE-Bra $\left(4.5^{\circ} \mathrm{E}, 51.3^{\circ} \mathrm{N}\right)$

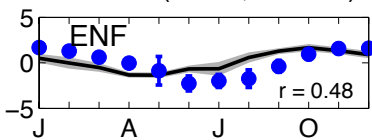

$\mathrm{CA}-\mathrm{Ca} 2\left(125.3^{\circ} \mathrm{W}, 49.9^{\circ} \mathrm{N}\right)$

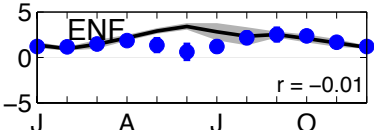

CA-NS1 $\left(98.5^{\circ} \mathrm{W}, 55.9^{\circ} \mathrm{N}\right)$

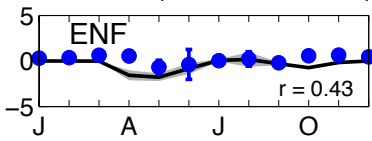

CA-NS5 $\left(98.5^{\circ} \mathrm{W}, 55.9^{\circ} \mathrm{N}\right)$

5 'ENF

oraceroreacen

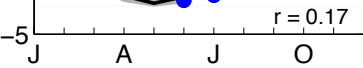

CA-Qfo $\left(74.3^{\circ} \mathrm{W}, 49.7^{\circ} \mathrm{N}\right)$

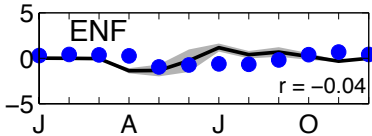

CA-TP4 $\left(80.4^{\circ} \mathrm{W}, 42.7^{\circ} \mathrm{N}\right)$
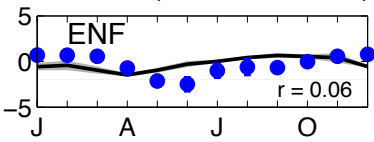

DE-Wet $\left(11.5^{\circ} \mathrm{E}, 50.5^{\circ} \mathrm{N}\right)$

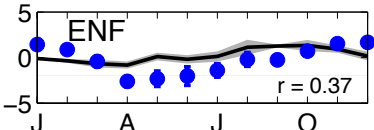

$\mathrm{FR}-\mathrm{LBr}\left(0.8^{\circ} \mathrm{W}, 44.7^{\circ} \mathrm{N}\right)$

5 'ENF

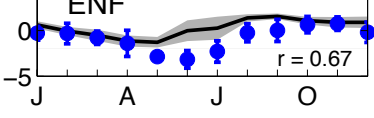

IT-SRo $\left(10.3^{\circ} \mathrm{E}, 43.7^{\circ} \mathrm{N}\right)$

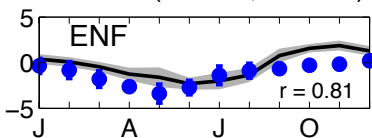

SE-Nor $\left(17.5^{\circ} \mathrm{E}, 60.1^{\circ} \mathrm{N}\right)$

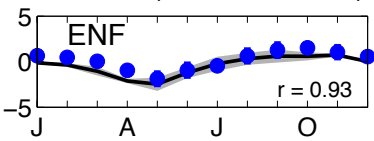

BE-Jal $\left(6.1^{\circ} \mathrm{E}, 50.6^{\circ} \mathrm{N}\right)$

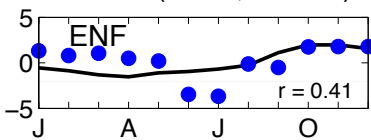

$\mathrm{CA}-\mathrm{Ca} 3\left(124.9^{\circ} \mathrm{W}, 49.5^{\circ} \mathrm{N}\right)$

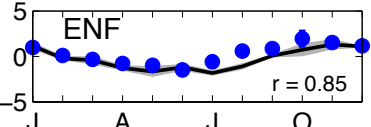

CA-NS2 $\left(98.5^{\circ} \mathrm{W}, 55.9^{\circ} \mathrm{N}\right)$

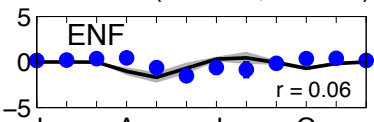

CA-Obs $\left(105.1^{\circ} \mathrm{W}, 54^{\circ} \mathrm{N}\right)$
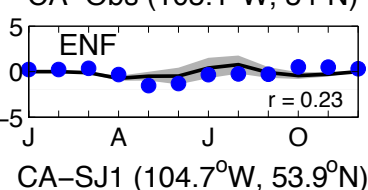

5 ENF

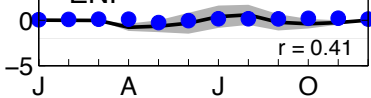

$\mathrm{CZ}-\mathrm{BK} 1\left(18.5^{\circ} \mathrm{E}, 49.5^{\circ} \mathrm{N}\right)$

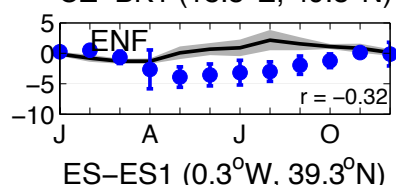

ES-ES1 $\left(0.3^{\circ} \mathrm{W}, 39.3^{\circ} \mathrm{N}\right)$

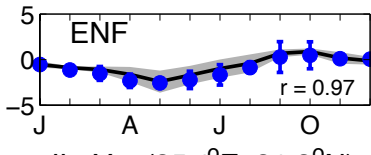

IL-Yat $\left(35.1^{\circ} \mathrm{E}, 31.3^{\circ} \mathrm{N}\right)$

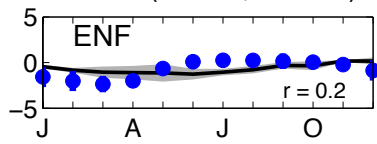

$\mathrm{NL}-$ Loo $\left(5.7^{\circ} \mathrm{E}, 52.2^{\circ} \mathrm{N}\right)$

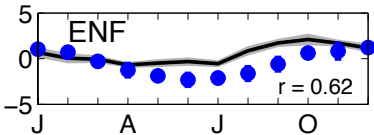

SE-Sk1 $\left(17.9^{\circ} \mathrm{E}, 60.1^{\circ} \mathrm{N}\right)$

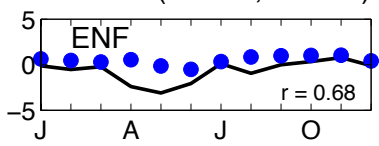

BE-Vie $\left(6^{\circ} \mathrm{E}, 50.3^{\circ} \mathrm{N}\right)$

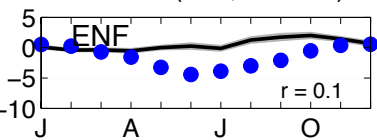

CA-Gro $\left(82.2^{\circ} \mathrm{W}, 48.2^{\circ} \mathrm{N}\right)$

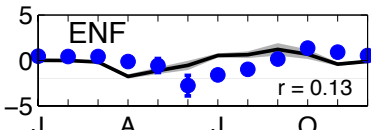

CA-NS3 $\left(98.4^{\circ} \mathrm{W}, 55.9^{\circ} \mathrm{N}\right)$

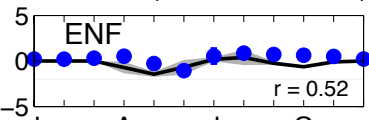

CA-Ojp $\left(104.7^{\circ} \mathrm{W}, 53.9^{\circ} \mathrm{N}\right)$

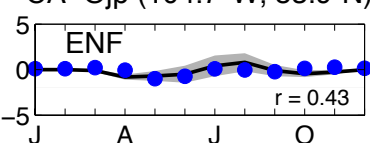

CA-SJ2 $\left(104.6^{\circ} \mathrm{W}, 53.9^{\circ} \mathrm{N}\right)$

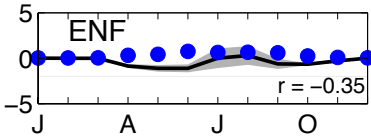

DE-Bay $\left(11.9^{\circ} \mathrm{E}, 50.1^{\circ} \mathrm{N}\right)$

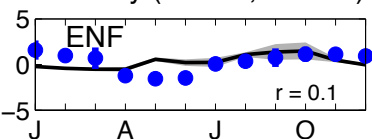

FI-Hyy $\left(24.3^{\circ} \mathrm{E}, 61.8^{\circ} \mathrm{N}\right)$

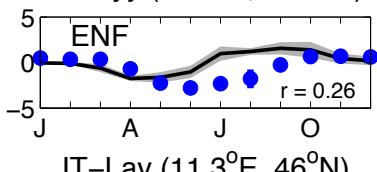

IT-Lav $\left(11.3^{\circ} \mathrm{E}, 46^{\circ} \mathrm{N}\right)$

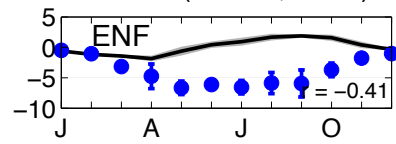

RU-Fyo $\left(32.9^{\circ} \mathrm{E}, 56.5^{\circ} \mathrm{N}\right)$

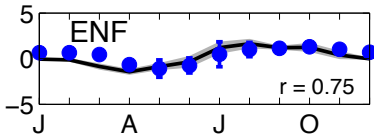

SE-Sk2 $\left(17.8^{\circ} \mathrm{E}, 60.1^{\circ} \mathrm{N}\right)$

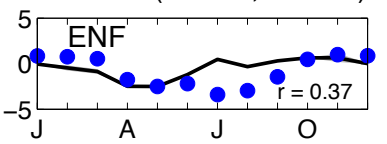

$\mathrm{CA}-\mathrm{Ca} 1\left(125.3^{\circ} \mathrm{W}, 49.9^{\circ} \mathrm{N}\right)$

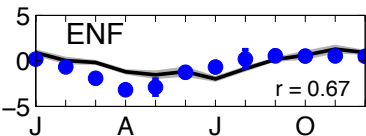

CA-Man $\left(98.5^{\circ} \mathrm{W}, 55.9^{\circ} \mathrm{N}\right)$

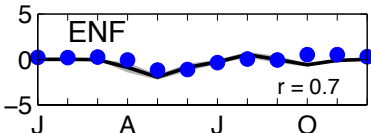

CA-NS4 $\left(98.4^{\circ} \mathrm{W}, 55.9^{\circ} \mathrm{N}\right)$

5 'ENF'

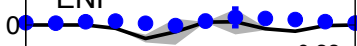

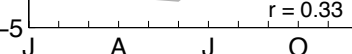

CA-Qcu $\left(74^{\circ} \mathrm{W}, 49.3^{\circ} \mathrm{N}\right)$

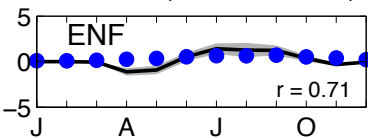

CA-SJ3 $\left(104.6^{\circ} \mathrm{W}, 53.9^{\circ} \mathrm{N}\right)$

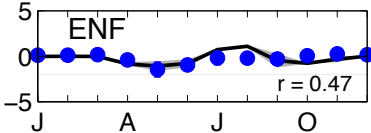

DE-Tha $\left(13.6^{\circ} \mathrm{E}, 51^{\circ} \mathrm{N}\right)$

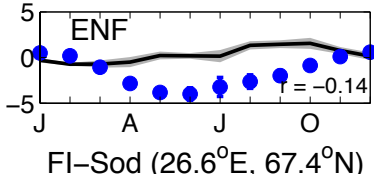

FI-Sod $\left(26.6^{\circ} \mathrm{E}, 67.4^{\circ} \mathrm{N}\right)$

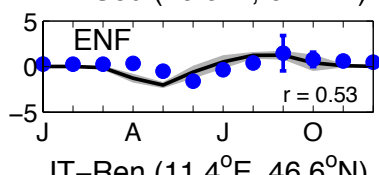

IT-Ren $\left(11.4^{\circ} \mathrm{E}, 46.6^{\circ} \mathrm{N}\right)$

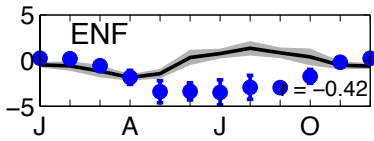

SE-Fla $\left(19.5^{\circ} \mathrm{E}, 64.1^{\circ} \mathrm{N}\right)$

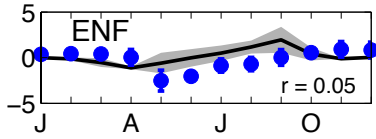

UK-Gri $\left(3.8^{\circ} \mathrm{W}, 56.6^{\circ} \mathrm{N}\right)$

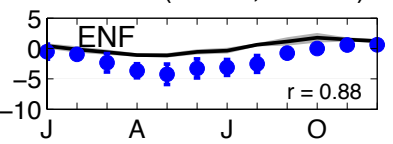

Figure S4. Comparison of simulated (solid lines) and observed (blue points) monthly net ecosystem exchange (NEE) at 145 sites from FLUXNET and NACP network. Both simulations and observations are averaged over the period when measurements are available (Table S1). Error bars represent one standard deviation of observations while shadings represent that of simulations. The relative bias and correlation coefficient are shown on each panel. The land types include evergreen needleleaf forest (ENF), evergreen broadleaf forest (EBF), deciduous broadleaf forest (DBF), shrublands (SHR), grasslands (GRA), and croplands (CRO). Units of NEE: $\mathrm{g} \mathrm{C} \mathrm{m}^{-2} \mathrm{day}^{-1}$. 


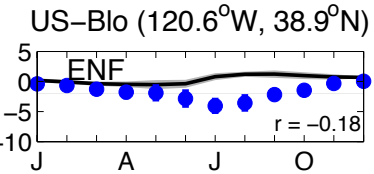

US-Me2 $\left(121.6^{\circ} \mathrm{W}, 44.5^{\circ} \mathrm{N}\right)$

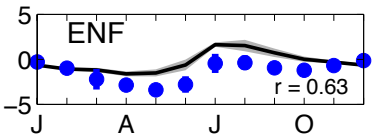

US-NR1 $\left(105.5^{\circ} \mathrm{W}, 40^{\circ} \mathrm{N}\right)$

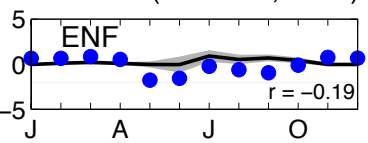

US-SP3 $\left(82.2^{\circ} \mathrm{W}, 29.8^{\circ} \mathrm{N}\right)$

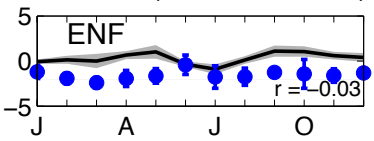

BR-Sa3 $\left(55^{\circ} \mathrm{W}, 3^{\circ} \mathrm{S}\right)$

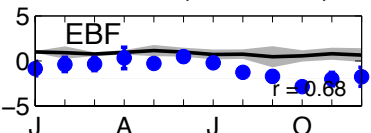

IT-Lec $\left(11.3^{\circ} \mathrm{E}, 43.3^{\circ} \mathrm{N}\right)$

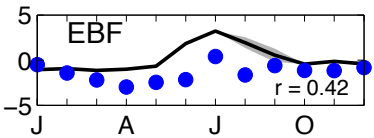

DE-Hai $\left(10.5^{\circ} \mathrm{E}, 51.1^{\circ} \mathrm{N}\right)$

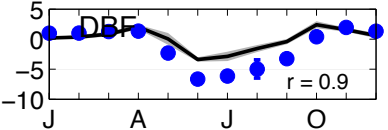

IT-Col $\left(13.6^{\circ} \mathrm{E}, 41.8^{\circ} \mathrm{N}\right)$

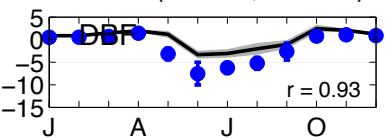

IT-Ro2 $\left(11.9^{\circ} \mathrm{E}, 42.4^{\circ} \mathrm{N}\right)$

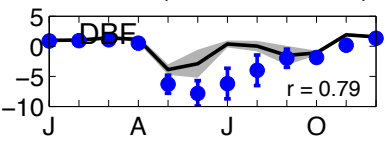

US-Ha1 $\left(72.2^{\circ} \mathrm{W}, 42.5^{\circ} \mathrm{N}\right)$

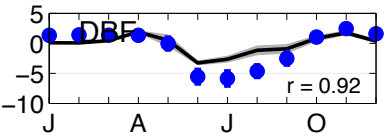

US-Dk3 $\left(79.1^{\circ} \mathrm{W}, 36^{\circ} \mathrm{N}\right)$

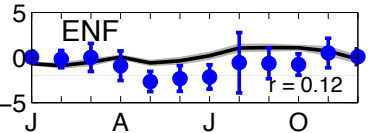

US-Me3 $\left(121.6^{\circ} \mathrm{W}, 44.3^{\circ} \mathrm{N}\right)$

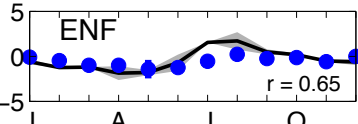

US-PFa $\left(90.3^{\circ} \mathrm{W}, 45.9^{\circ} \mathrm{N}\right)$

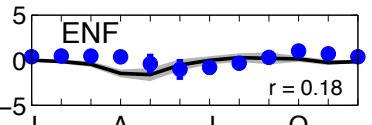

US-Syv $\left(89.3^{\circ} \mathrm{W}, 46.2^{\circ} \mathrm{N}\right)$

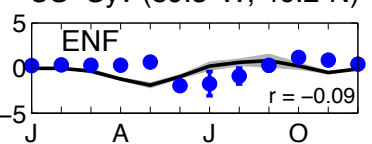

FR-Pue $\left(3.6^{\circ} \mathrm{E}, 43.7^{\circ} \mathrm{N}\right)$

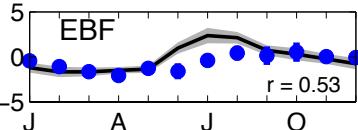

PT-Esp $\left(8.6^{\circ} \mathrm{W}, 38.6^{\circ} \mathrm{N}\right)$

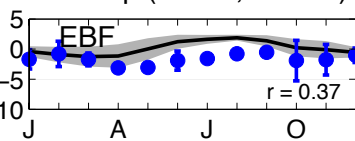

DK-Sor $\left(11.6^{\circ} \mathrm{E}, 55.5^{\circ} \mathrm{N}\right)$

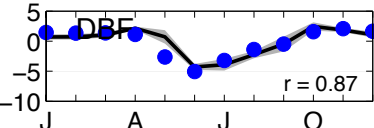

IT-Non $\left(11.1^{\circ} \mathrm{E}, 44.7^{\circ} \mathrm{N}\right)$

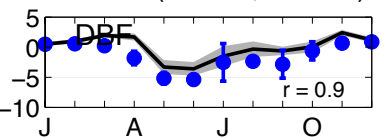

UK-Ham $\left(0.9^{\circ} \mathrm{W}, 51.2^{\circ} \mathrm{N}\right)$

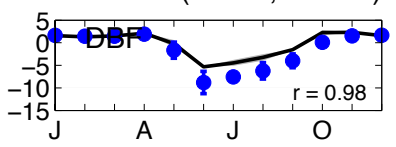

US-MMS $\left(86.4^{\circ} \mathrm{W}, 39.3^{\circ} \mathrm{N}\right)$

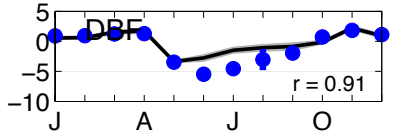

US-Ho1 $\left(68.7^{\circ} \mathrm{W}, 45.2^{\circ} \mathrm{N}\right)$

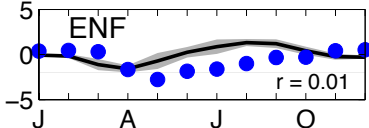

US-Me4 $\left(121.6^{\circ} \mathrm{W}, 44.5^{\circ} \mathrm{N}\right)$

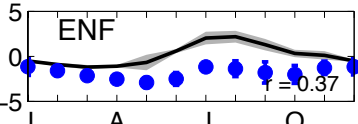

US-SP1 $\left(82.2^{\circ} \mathrm{W}, 29.7^{\circ} \mathrm{N}\right)$

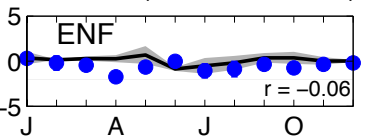

AU-Tum (148.2 $\left.\mathrm{E}, 35.7^{\circ} \mathrm{S}\right)$

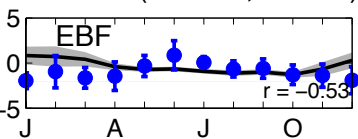

ID-Pag $\left(114^{\circ} \mathrm{E}, 2.3^{\circ} \mathrm{N}\right)$

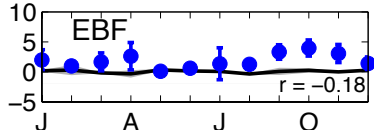

PT-Mi1 $\left(8^{\circ} \mathrm{W}, 38.5^{\circ} \mathrm{N}\right)$

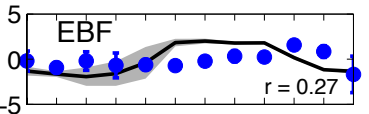

FR-Fon $\left(2.8^{\circ} \mathrm{E}, 48.5^{\circ} \mathrm{N}\right)$

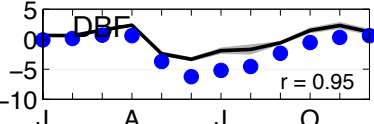

IT-PT1 $\left(9.1^{\circ} \mathrm{E}, 45.2^{\circ} \mathrm{N}\right)$

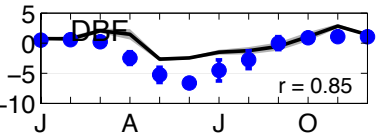

UK-PL3 $\left(1.3^{\circ} \mathrm{W}, 51.5^{\circ} \mathrm{N}\right)$

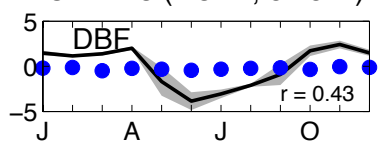

US-MOz $\left(92.2^{\circ} \mathrm{W}, 38.7^{\circ} \mathrm{N}\right)$

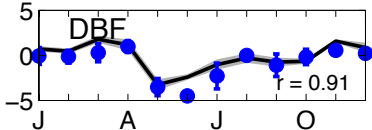

US-Ho2 $\left(68.7^{\circ} \mathrm{W}, 45.2^{\circ} \mathrm{N}\right)$

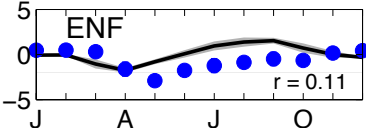

US-Me5 $\left(121.6^{\circ} \mathrm{W}, 44.4^{\circ} \mathrm{N}\right)$

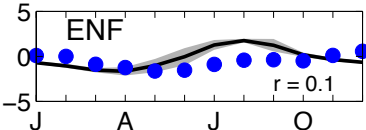

US-SP2 $\left(82.2^{\circ} \mathrm{W}, 29.8^{\circ} \mathrm{N}\right)$

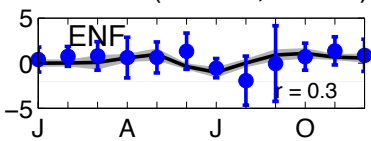

$\mathrm{AU}-\mathrm{Wac}\left(145.2^{\circ} \mathrm{E}, 37.4^{\circ} \mathrm{S}\right)$

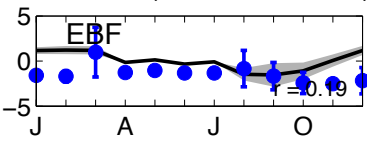

IT-Cpz $\left(12.4^{\circ} \mathrm{E}, 41.7^{\circ} \mathrm{N}\right)$

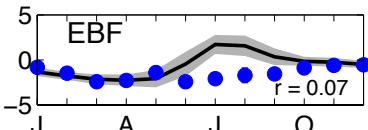

CA-Oas $\left(106.2^{\circ} \mathrm{W}, 53.6^{\circ} \mathrm{N}\right)$

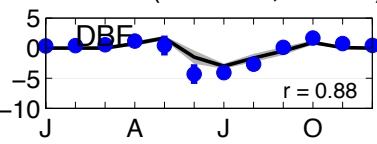

FR-Hes $\left(7.1^{\circ} \mathrm{E}, 48.7^{\circ} \mathrm{N}\right)$

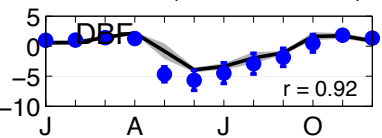

IT-Ro1 $\left(11.9^{\circ} \mathrm{E}, 42.4^{\circ} \mathrm{N}\right)$

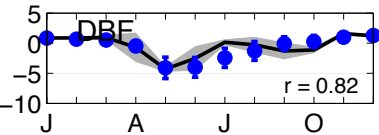

US-Bar $\left(71.3^{\circ} \mathrm{W}, 44.1^{\circ} \mathrm{N}\right)$

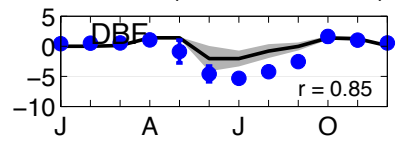

US-Oho $\left(83.8^{\circ} \mathrm{W}, 41.6^{\circ} \mathrm{N}\right)$

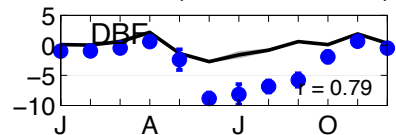

Figure S4. Continued. 


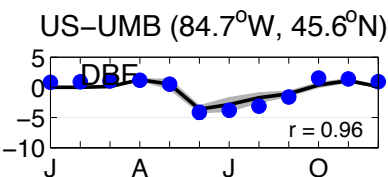

$\mathrm{AU}-\operatorname{How}\left(131.2^{\circ} \mathrm{E}, 12.5^{\circ} \mathrm{S}\right)$

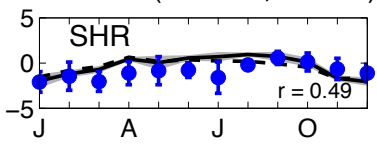

CA-NS7 $\left(99.9^{\circ} \mathrm{W}, 56.6^{\circ} \mathrm{N}\right)$

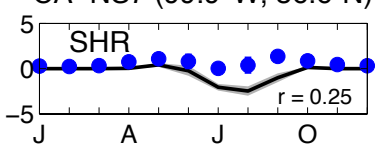

IT-Pia $\left(10.1^{\circ} \mathrm{E}, 42.6^{\circ} \mathrm{N}\right)$

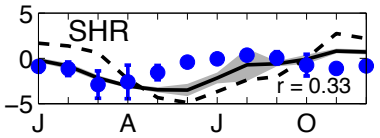

UK-AMo $\left(3.2^{\circ} \mathrm{W}, 55.8^{\circ} \mathrm{N}\right)$

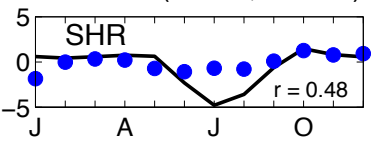

ZA-Kru $\left(31.5^{\circ} \mathrm{E}, 25^{\circ} \mathrm{S}\right)$
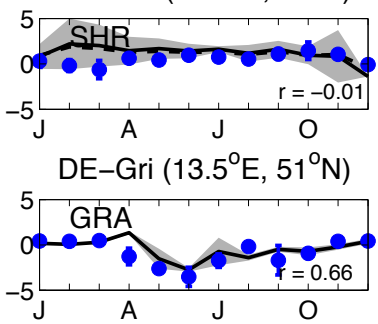

FR-Lq1 $\left(2.7^{\circ} \mathrm{E}, 45.6^{\circ} \mathrm{N}\right)$

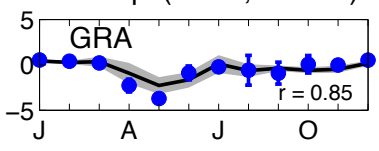

IE-Dri $\left(8.8^{\circ} \mathrm{W}, 52^{\circ} \mathrm{N}\right)$

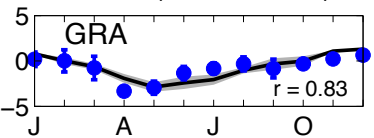

IT-Mal $\left(11.7^{\circ} \mathrm{E}, 46.1^{\circ} \mathrm{N}\right)$

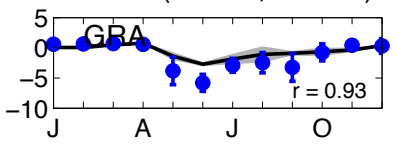

US-WBW $\left(84.3^{\circ} \mathrm{W}, 36^{\circ} \mathrm{N}\right)$

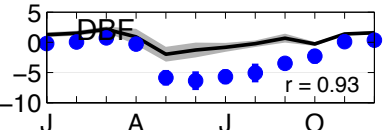

BW-Ma1 $\left(23.6^{\circ} \mathrm{E}, 19.9^{\circ} \mathrm{S}\right)$

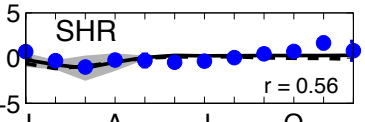

CA-WP1 $\left(112.5^{\circ} \mathrm{W}, 55^{\circ} \mathrm{N}\right)$

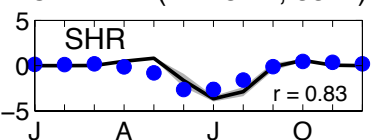

PL-wet $\left(16.3^{\circ} \mathrm{E}, 52.8^{\circ} \mathrm{N}\right)$

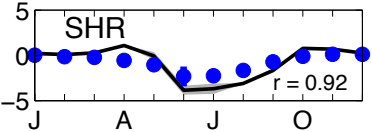

US-Los $\left(90^{\circ} \mathrm{W}, 46.1^{\circ} \mathrm{N}\right)$

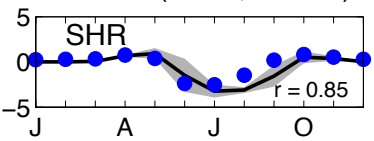

AT-Neu $\left(11.3^{\circ} \mathrm{E}, 47.1^{\circ} \mathrm{N}\right)$

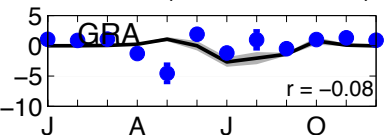

DE-Meh $\left(10.7^{\circ} \mathrm{E}, 51.3^{\circ} \mathrm{N}\right)$

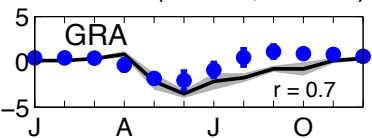

FR-Lq2 $\left(2.7^{\circ} \mathrm{E}, 45.6^{\circ} \mathrm{N}\right)$

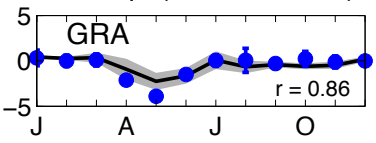

IT-Amp $\left(13.6^{\circ} \mathrm{E}, 41.9^{\circ} \mathrm{N}\right)$

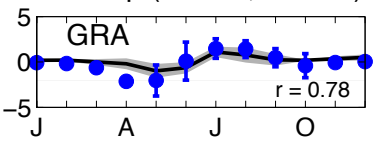

$\mathrm{NL}-\mathrm{Ca} 1\left(4.9^{\circ} \mathrm{E}, 52^{\circ} \mathrm{N}\right)$

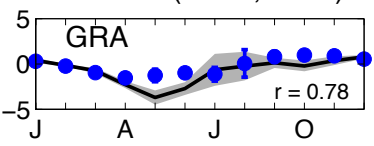

US-WCr $\left(90.1^{\circ} \mathrm{W}, 45.8^{\circ} \mathrm{N}\right)$

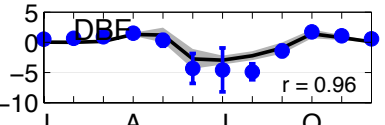

CA-Mer $\left(75.5^{\circ} \mathrm{W}, 45.4^{\circ} \mathrm{N}\right)$

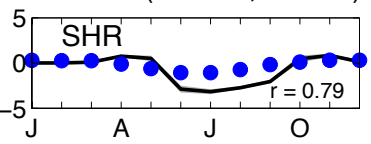

ES-LMa $\left(5.8^{\circ} \mathrm{W}, 39.9^{\circ} \mathrm{N}\right)$

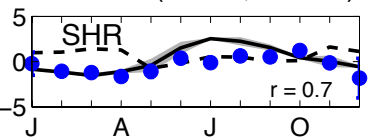

SE-Deg $\left(19.6^{\circ} \mathrm{E}, 64.2^{\circ} \mathrm{N}\right)$

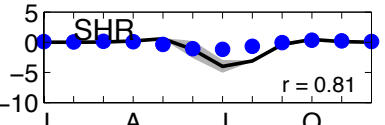

US-SO2 $\left(116.6^{\circ} \mathrm{W}, 33.4^{\circ} \mathrm{N}\right)$

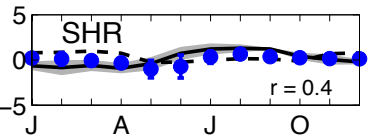

CA-Let $\left(112.9^{\circ} \mathrm{W}, 49.7^{\circ} \mathrm{N}\right)$

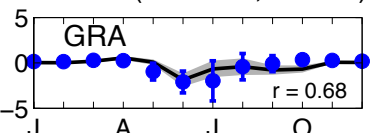

DK-Lva $\left(12.1^{\circ} \mathrm{E}, 55.7^{\circ} \mathrm{N}\right)$

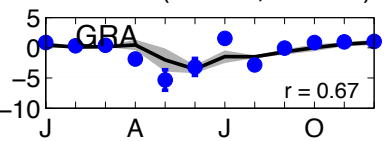

HU-Bug $\left(19.6^{\circ} \mathrm{E}, 46.7^{\circ} \mathrm{N}\right)$

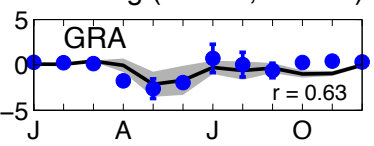

IT-LMa $\left(7.2^{\circ} \mathrm{E}, 45.6^{\circ} \mathrm{N}\right)$

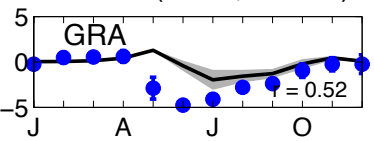

$\mathrm{NL}-\mathrm{Hor}\left(5.1^{\circ} \mathrm{E}, 52^{\circ} \mathrm{N}\right)$

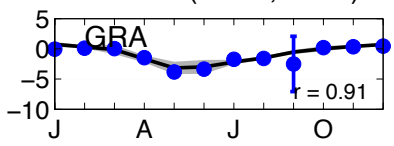

$A U-F o g\left(131.3^{\circ} \mathrm{E}, 12.5^{\circ} \mathrm{S}\right)$

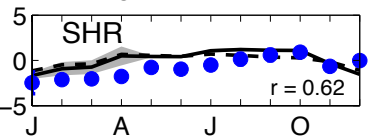

CA-NS6 $\left(99^{\circ} \mathrm{W}, 55.9^{\circ} \mathrm{N}\right)$

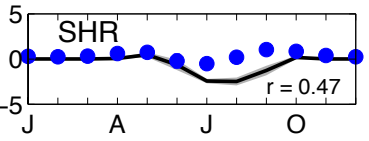

$\mathrm{FI}-\mathrm{Kaa}\left(27.3^{\circ} \mathrm{E}, 69.1^{\circ} \mathrm{N}\right)$

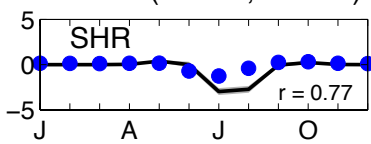

SE-Faj $\left(13.6^{\circ} \mathrm{E}, 56.3^{\circ} \mathrm{N}\right)$

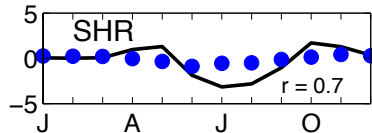

US-Ton $\left(121^{\circ} \mathrm{W}, 38.4^{\circ} \mathrm{N}\right)$

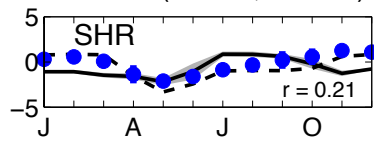

$\mathrm{CH}-\mathrm{Oe} 1\left(7.7^{\circ} \mathrm{E}, 47.3^{\circ} \mathrm{N}\right)$

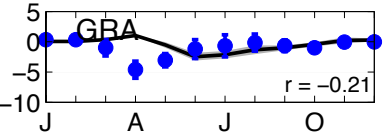

ES-VDA $\left(1.4^{\circ} \mathrm{E}, 42.2^{\circ} \mathrm{N}\right)$

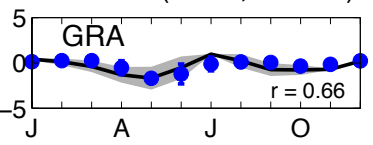

HU-Mat $\left(19.7^{\circ} \mathrm{E}, 47.8^{\circ} \mathrm{N}\right)$

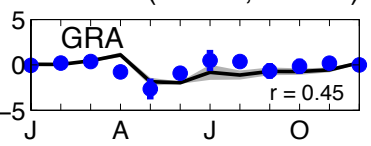

IT-MBo $\left(11^{\circ} \mathrm{E}, 46^{\circ} \mathrm{N}\right)$

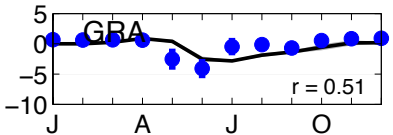

PT-Mi2 $\left(8^{\circ} \mathrm{W}, 38.5^{\circ} \mathrm{N}\right)$

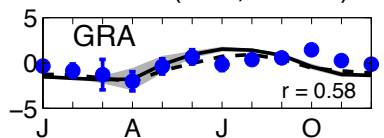

Figure S4. Continued. Dashed lines in some shrub and grass sites represent simulations with cold phenology alone. 

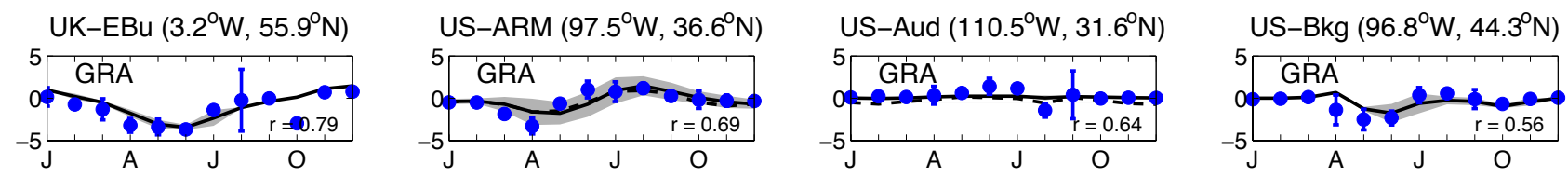

US-FPe $\left(105.1^{\circ} \mathrm{W}, 48.3^{\circ} \mathrm{N}\right)$
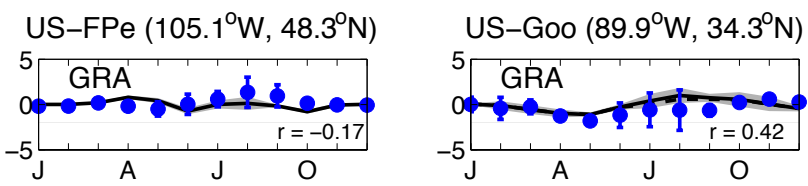

US-IB2 $\left(88.2^{\circ} \mathrm{W}, 41.8^{\circ} \mathrm{N}\right)$

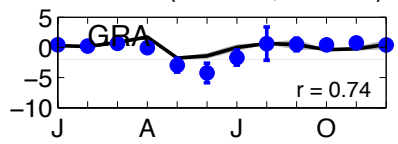

US-Shd $\left(96.7^{\circ} \mathrm{W}, 36.9^{\circ} \mathrm{N}\right)$

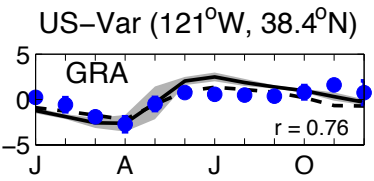

BE-Lon $\left(4.7^{\circ} \mathrm{E}, 50.6^{\circ} \mathrm{N}\right)$

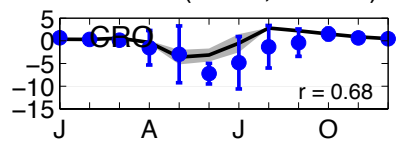

$\mathrm{CH}-\mathrm{Oe} 2\left(7.7^{\circ} \mathrm{E}, 47.3^{\circ} \mathrm{N}\right)$
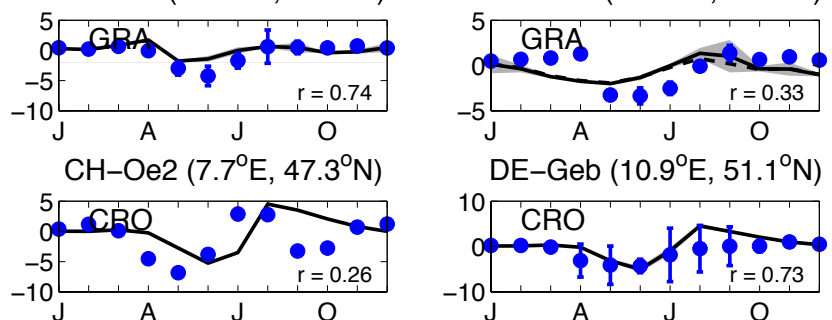

DE-Geb $\left(10.9^{\circ} \mathrm{E}, 51.1^{\circ} \mathrm{N}\right)$
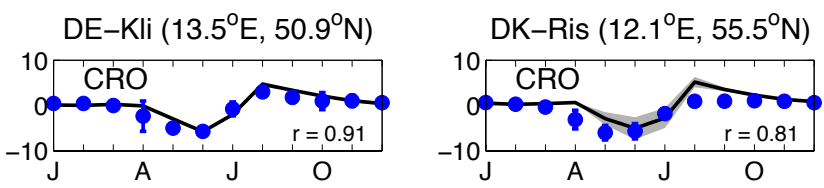

ES-ES2 $\left(0.3^{\circ} \mathrm{W}, 39.3^{\circ} \mathrm{N}\right)$
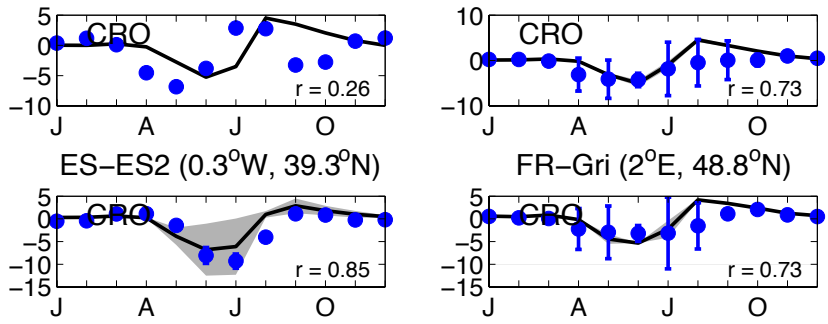

FR-Gri $\left(2^{\circ} \mathrm{E}, 48.8^{\circ} \mathrm{N}\right)$
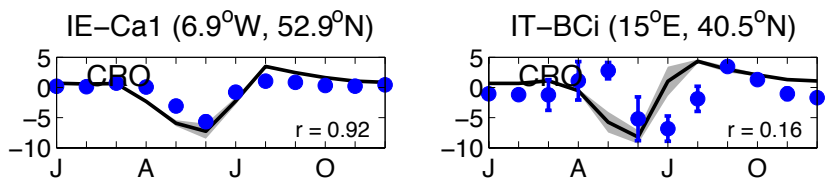

$\mathrm{NL}-\operatorname{Lan}\left(4.9^{\circ} \mathrm{E}, 52^{\circ} \mathrm{N}\right)$
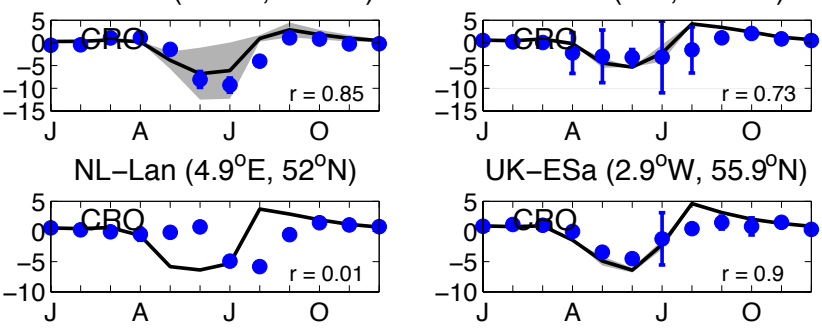

US-Bo1 $\left(88.3^{\circ} \mathrm{W}, 40^{\circ} \mathrm{N}\right)$

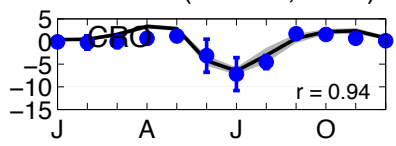

US-IB1 $\left(88.2^{\circ} \mathrm{W}, 41.9^{\circ} \mathrm{N}\right)$

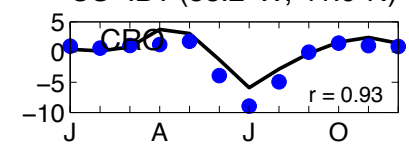

US-Ne1 $\left(96.5^{\circ} \mathrm{W}, 41.2^{\circ} \mathrm{N}\right)$
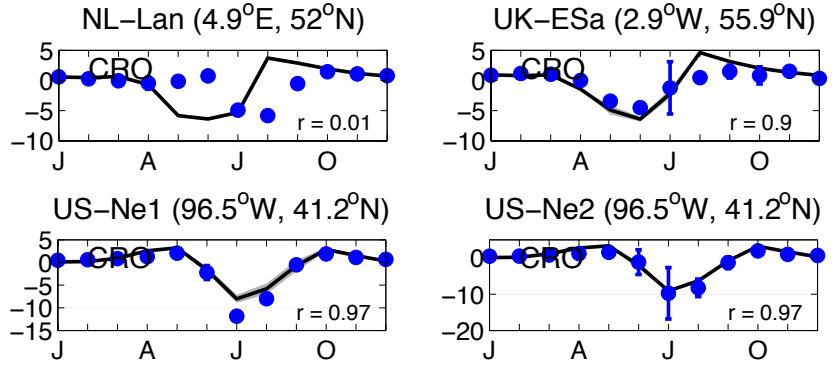

US-Ne2 $\left(96.5^{\circ} \mathrm{W}, 41.2^{\circ} \mathrm{N}\right)$

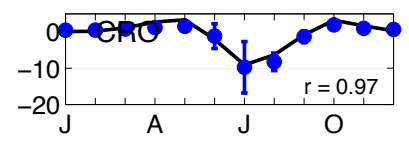

US-Ne3 $\left(96.4^{\circ} \mathrm{W}, 41.2^{\circ} \mathrm{N}\right)$

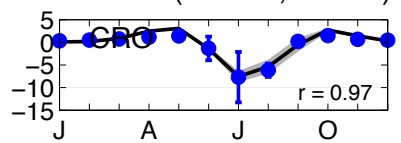

Figure S4. Continued. Dashed lines in some grass sites represent simulations with cold phenology alone. 
(a) Simulated annual surface [O3]

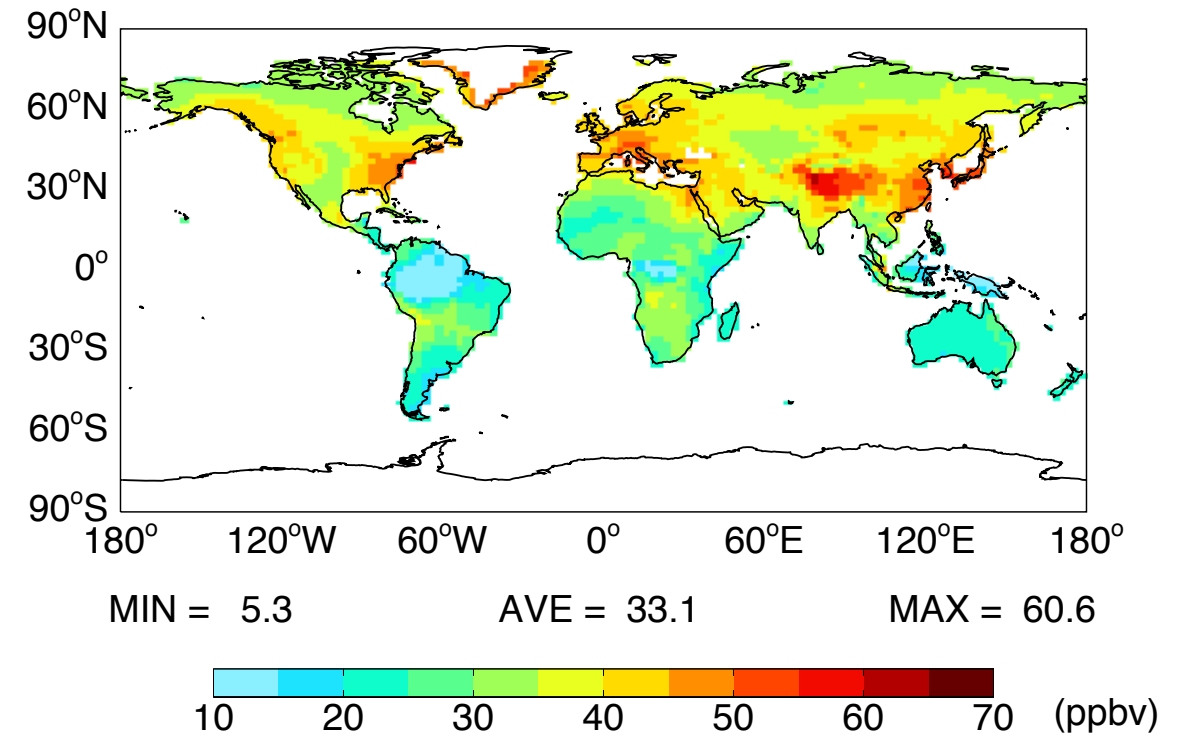

(b) Simulated JJA surface [O3]

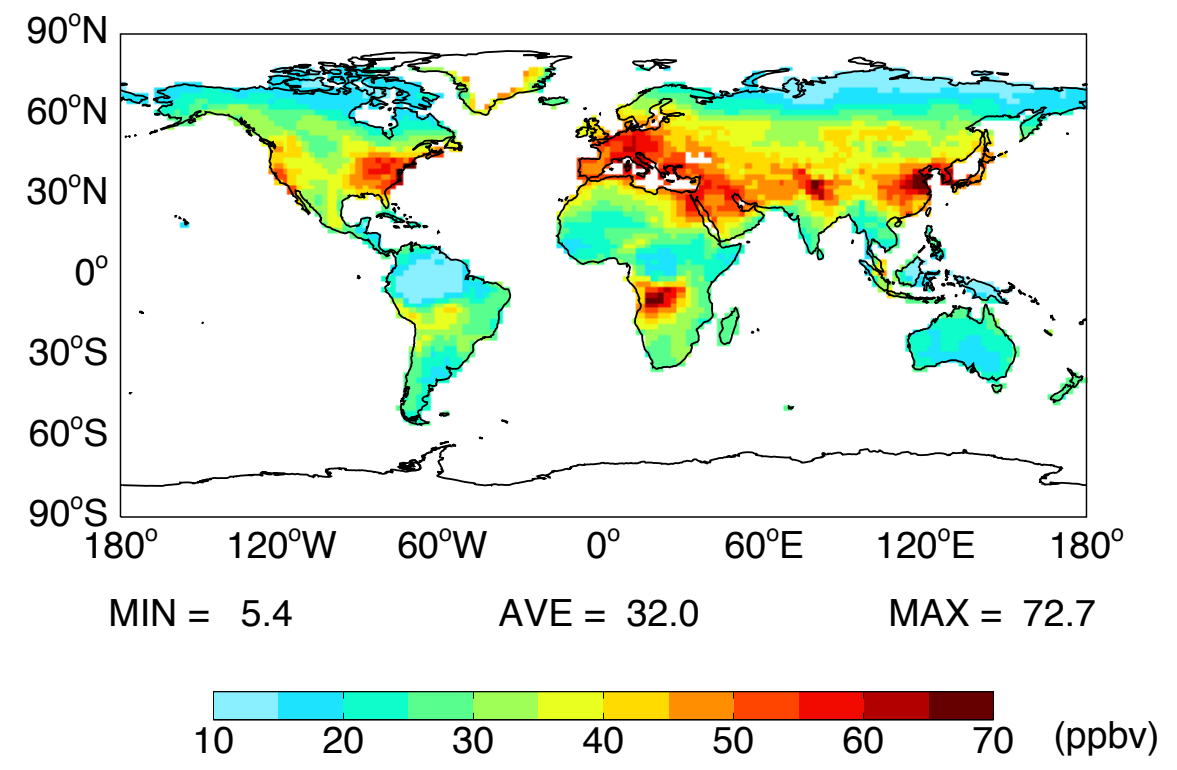

Figure S5. Simulated (a) annual and (b) June-August mean surface ozone concentrations with ModelE2. 
(a) JAN ModelE2-YIBS LAI (1.0)

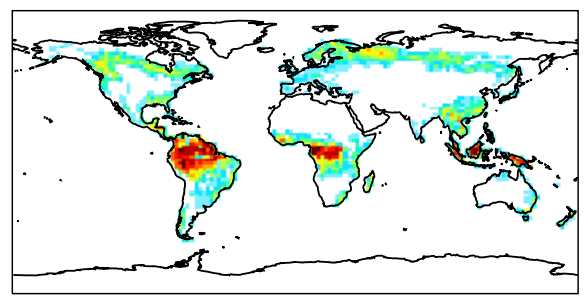

(c) APR ModelE2-YIBS LAI (1.0)

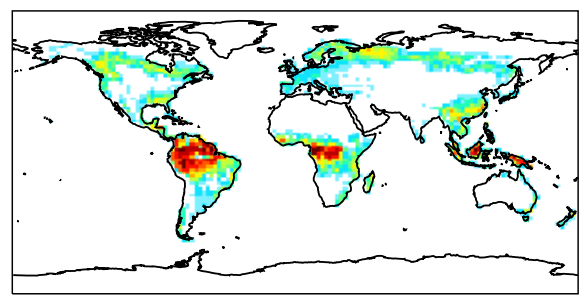

(e) JUL ModelE2-YIBS LAI (1.3)

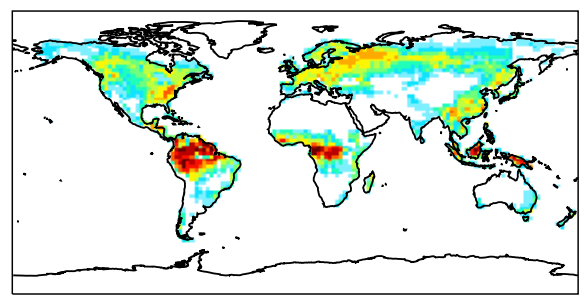

(g) OCT ModelE2-YIBS LAI (1.1)
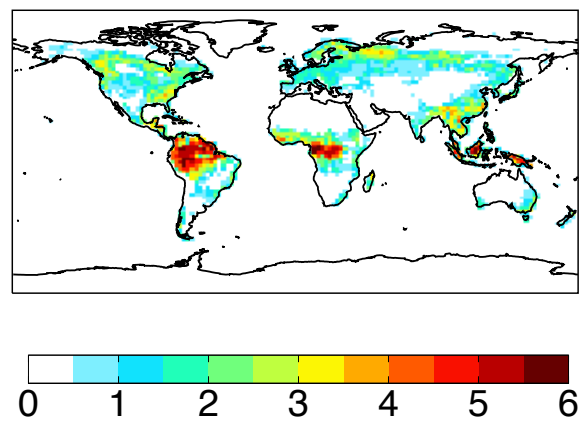

(b) JAN Diff: ModelE2 - Offline (-0.2)

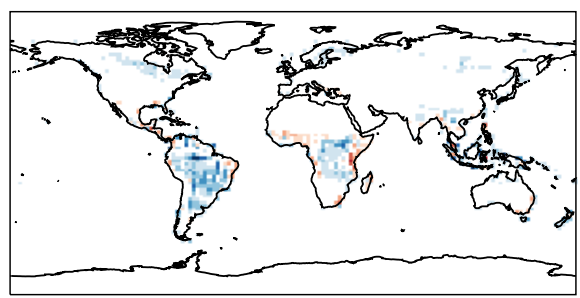

(d) APR Diff: ModelE2 - Offline (-0.2)

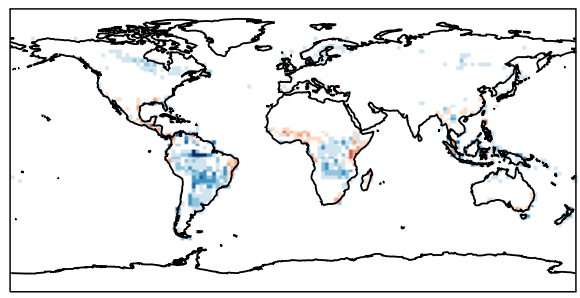

(f) JUL Diff: ModelE2 - Offline (-0.2)

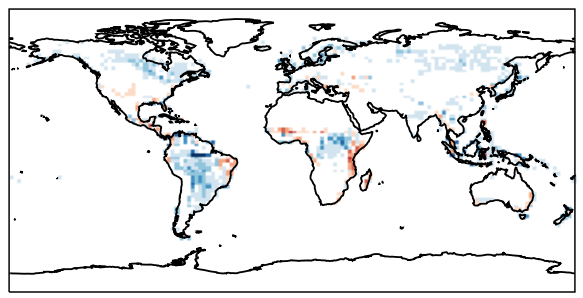

(h) OCT Diff: ModelE2 - Offline (-0.2)

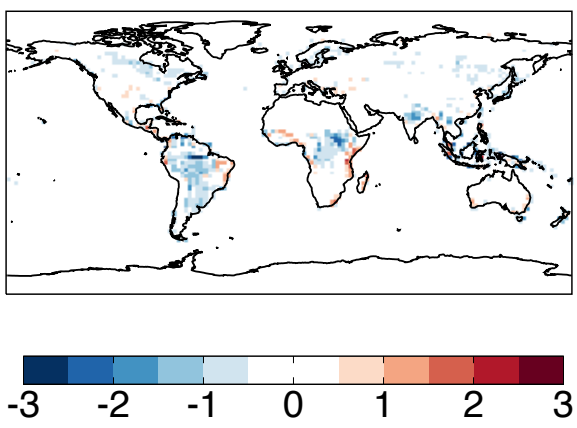

Figure S6. Simulated (left) leaf area index (LAI) with ModelE2-YIBs and (right) its differences relative to simulations with offline YIBs driven by WFDEI reanalysis for period 1996-2005. Each row represents results averaged for a specific month. Units: $\mathrm{m}^{2}$ $\mathrm{m}^{-2}$. 
(a) JAN ModelE2-YIBS GPP (1.7)

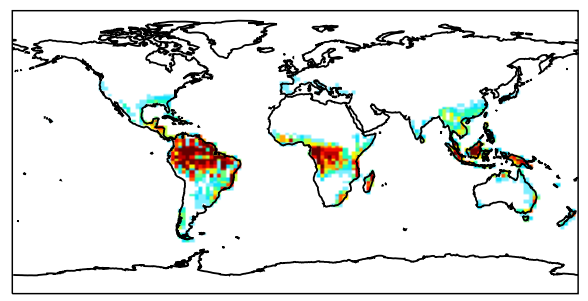

(c) APR ModelE2-YIBS GPP (2.0)

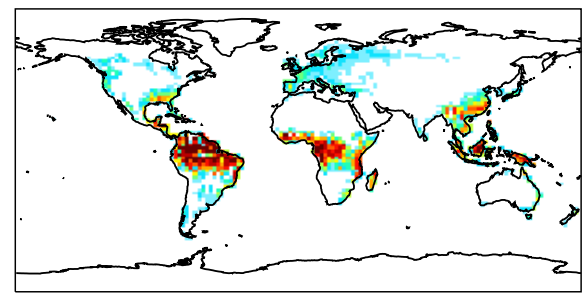

(e) JUL ModelE2-YIBS GPP (3.2)

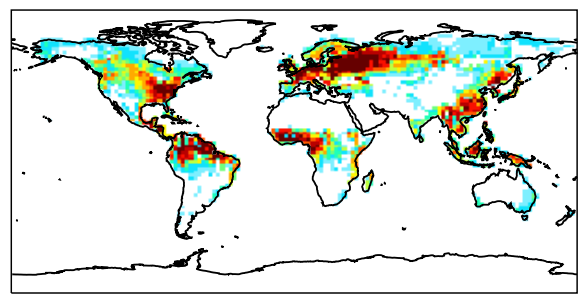

(g) OCT ModelE2-YIBS GPP (2.0)
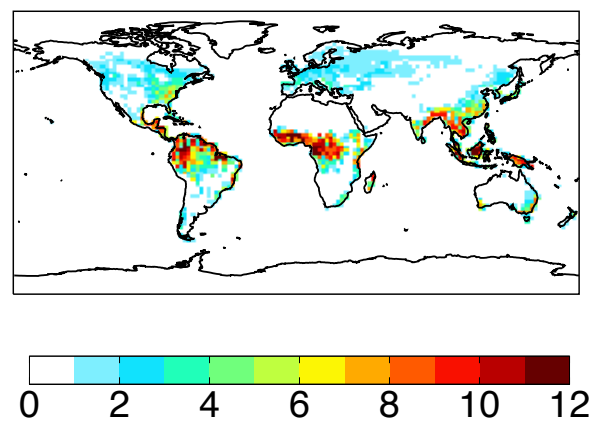

(b) JAN Diff: ModelE2 - Offline (-0.0)

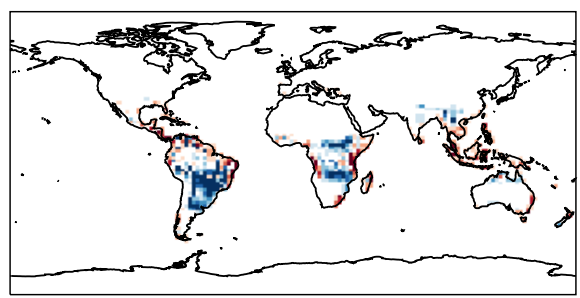

(d) APR Diff: ModelE2 - Offline ( 0.0)

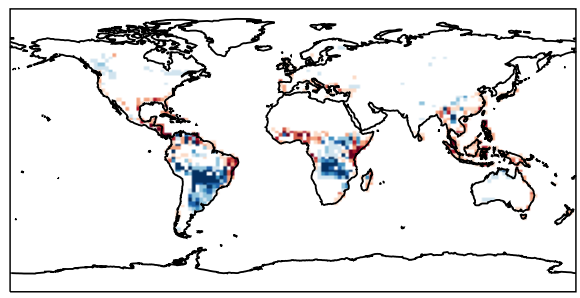

(f) JUL Diff: ModelE2 - Offline (-0.2)

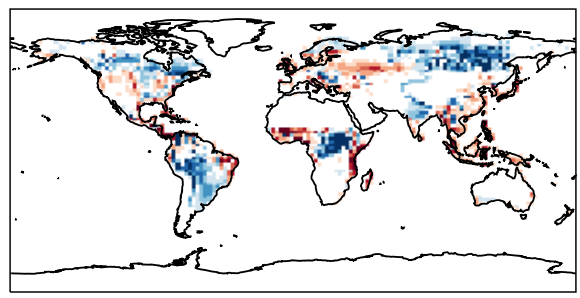

(h) OCT Diff: ModelE2 - Offline (-0.2)

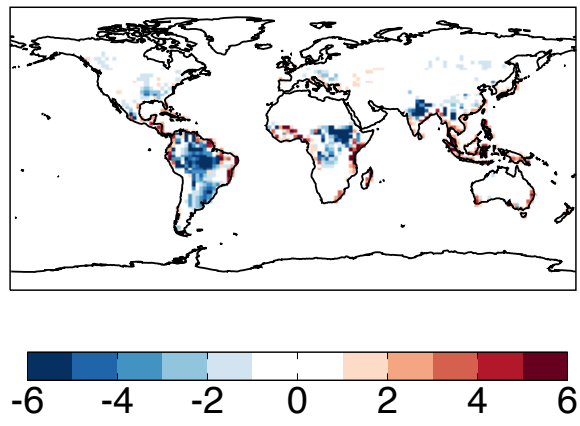

Figure S7. Simulated (left) GPP with ModelE2-YIBs and (right) its differences relative to simulations with offline YIBs driven by WFDEI reanalysis for period 1996-2005. Each row represents results averaged for a specific month. Units: $\mathrm{g} \mathrm{m}^{-2} \mathrm{day}^{-1}$. 
(a) JAN ModelE2-YIBS NPP (0.9)

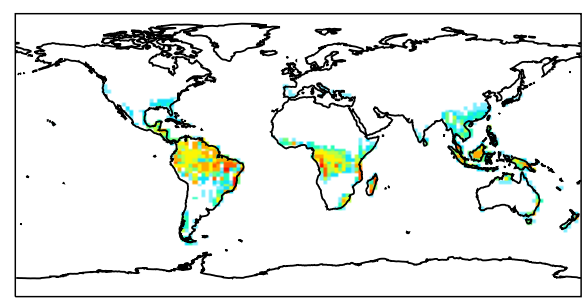

(c) APR ModelE2-YIBS NPP (1.0)

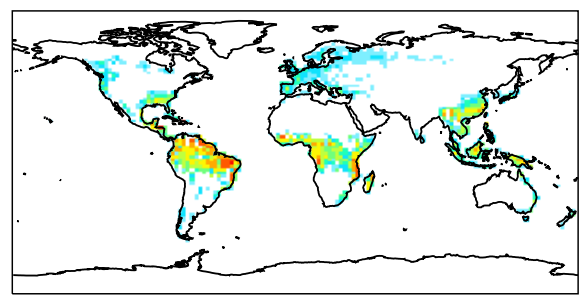

(e) JUL ModelE2-YIBS NPP (1.8)

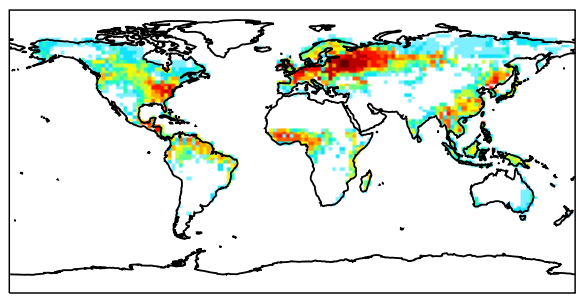

(g) OCT ModelE2-YIBS NPP (1.0)
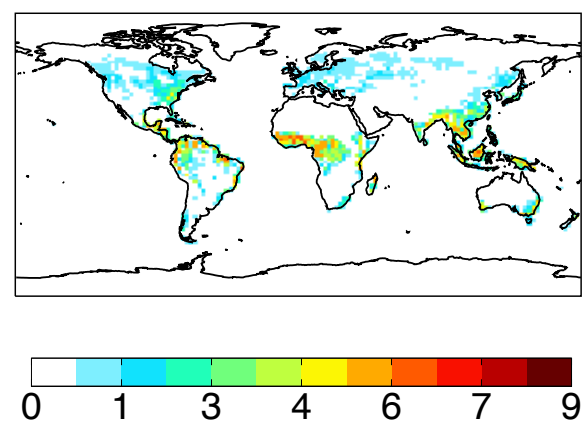

(b) JAN Diff: ModelE2 - Offline (-0.0)

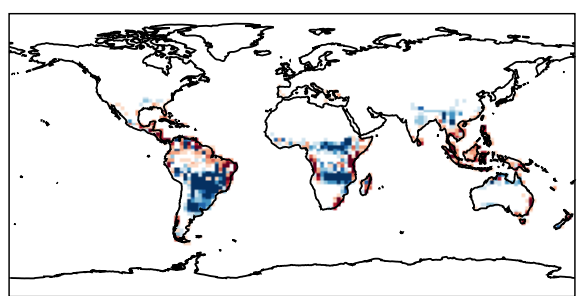

(d) APR Diff: ModelE2 - Offline (-0.0)

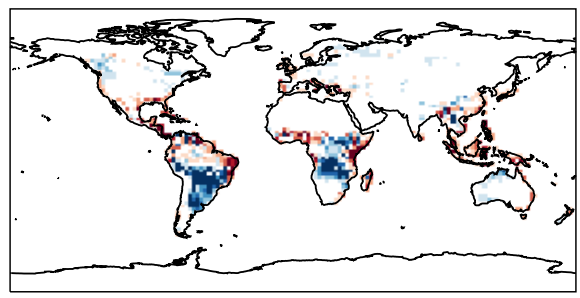

(f) JUL Diff: ModelE2 - Offline (-0.2)

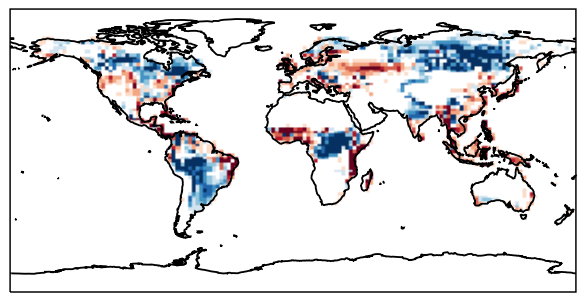

(h) OCT Diff: ModelE2 - Offline (-0.1)

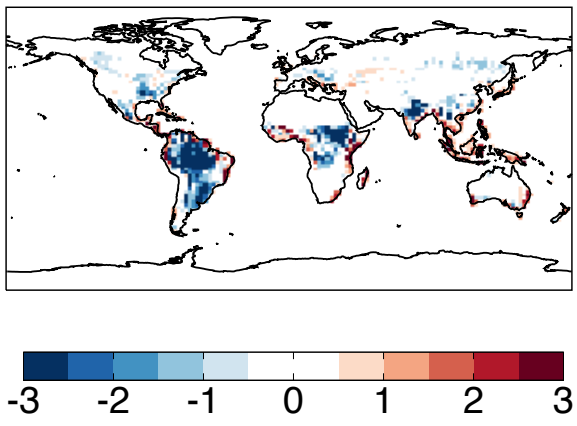

Figure S8. Simulated (left) net primary productivity (NPP) with ModelE2-YIBs and (right) its differences relative to simulations with offline YIBs driven by WFDEI reanalysis for period 1996-2005. Each row represents results averaged for a specific month. Units: $\mathrm{g} \mathrm{m}^{-2}$ day $^{-1}$. 

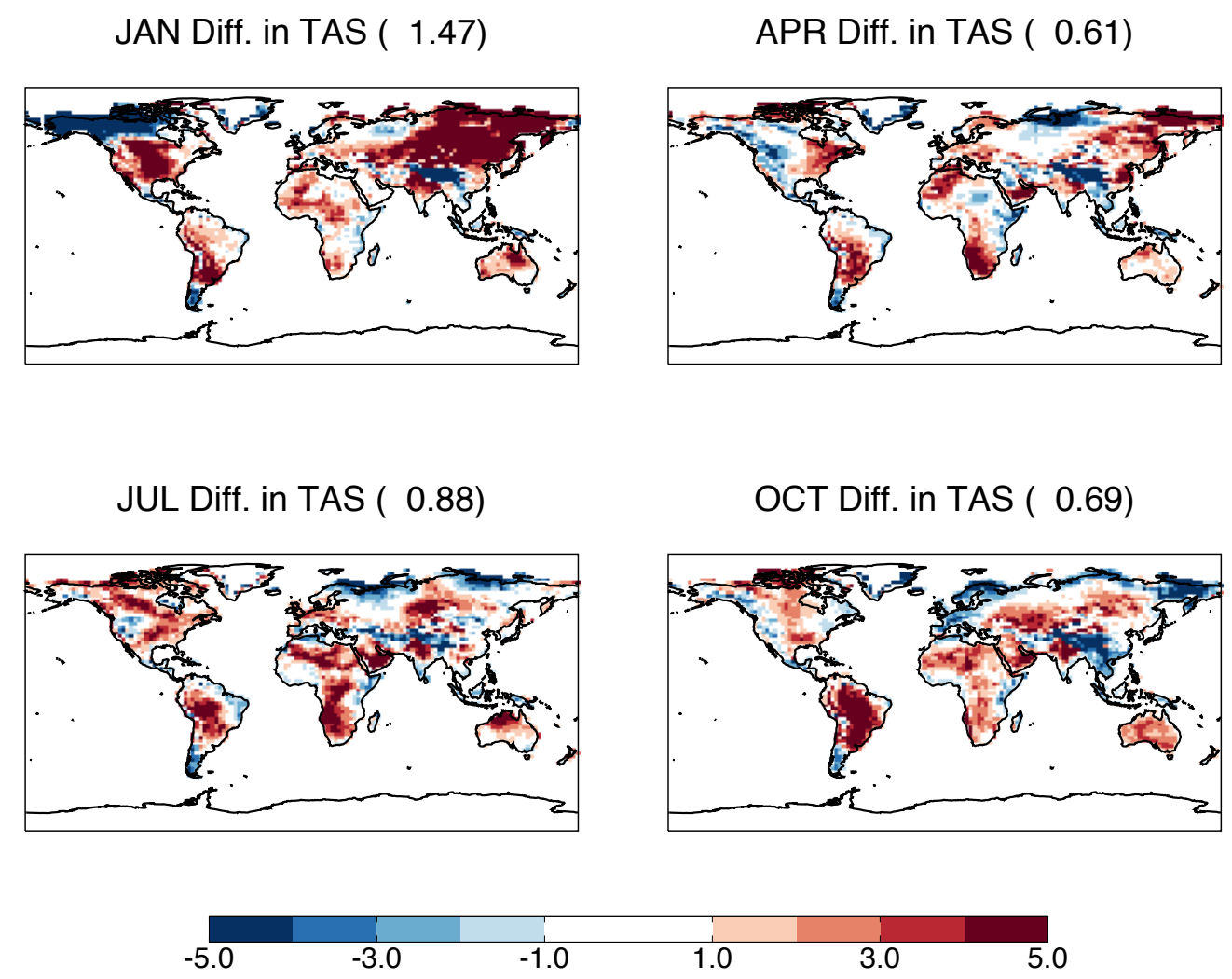

Figure S9. Differences of surface air temperature between ModelE2-YIBs and WFDEI for period 1996-2005. Each panel represents results averaged for a specific month. Units: ${ }^{\circ} \mathrm{C}$. 

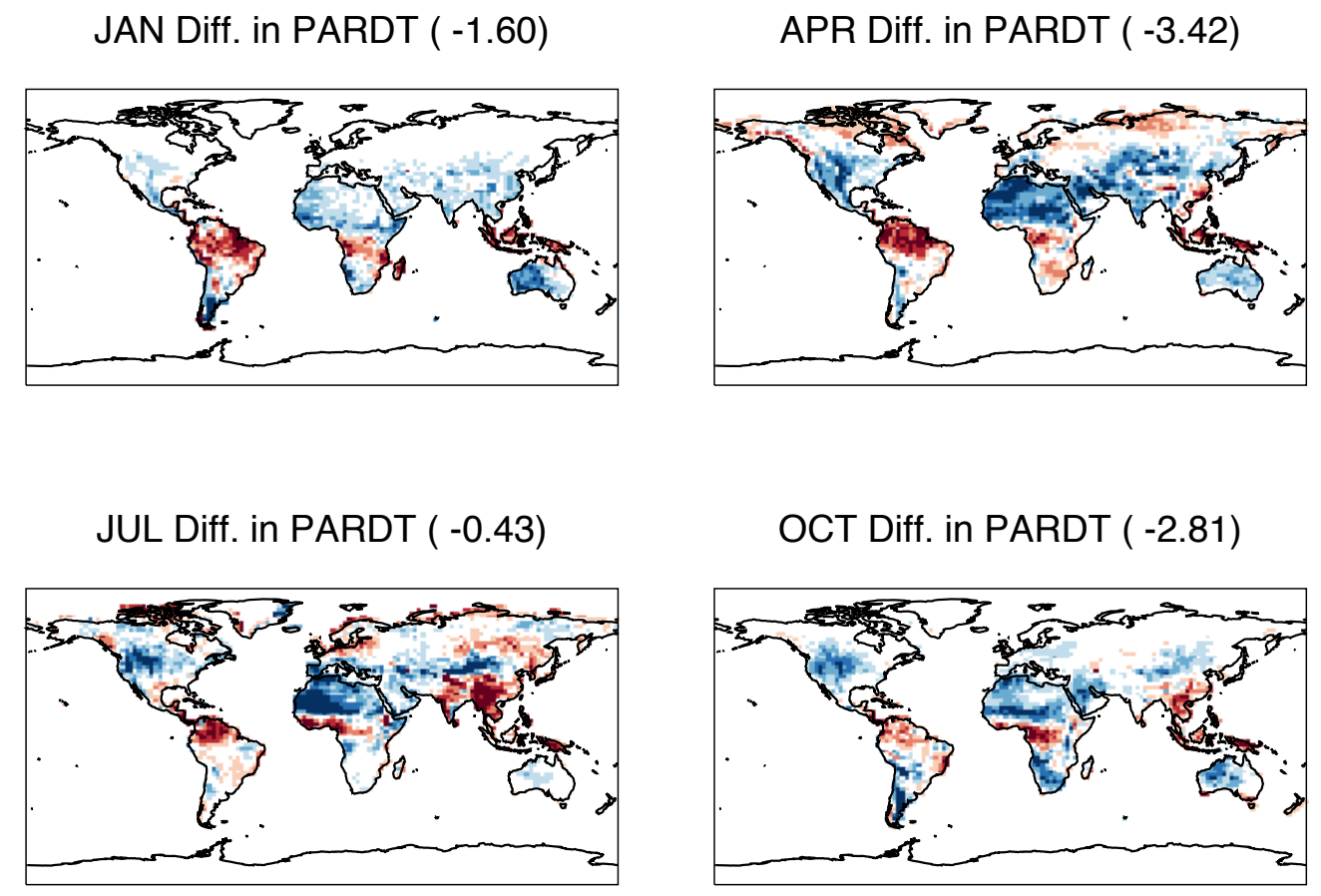

OCT Diff. in PARDT ( -2.81)
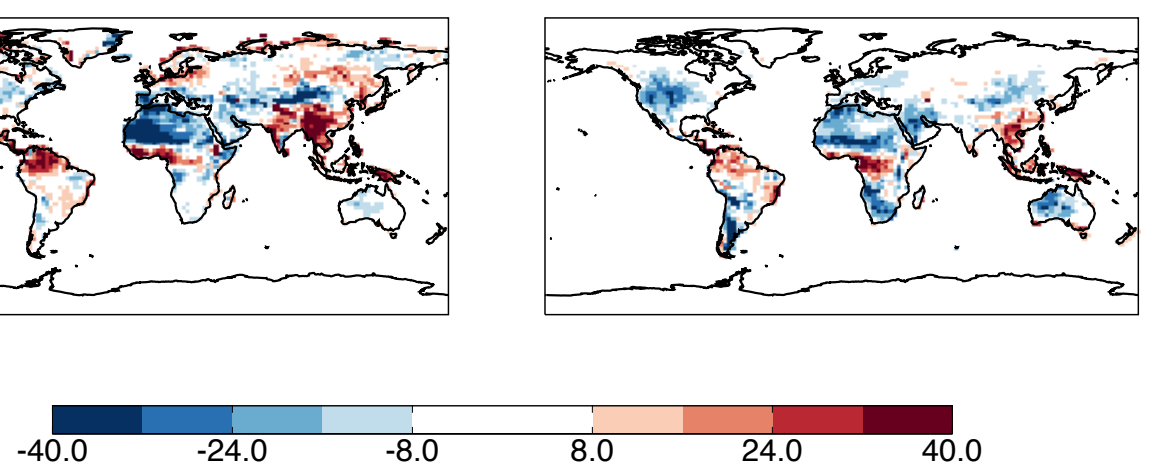

Figure S10. Differences of total Photosynthetically active radiation (PAR) between ModelE2-YIBs and WFDEI for period 1996-2005. Each panel represents results averaged for a specific month. Units: $\mathrm{W} \mathrm{m}^{-2}$. 
JAN Diff. in QCAN ( 0.15)

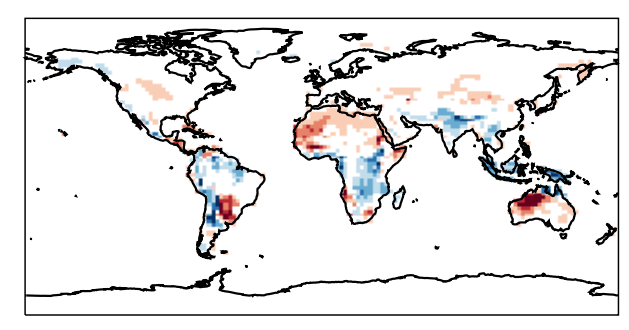

JUL Diff. in QCAN ( -0.25)

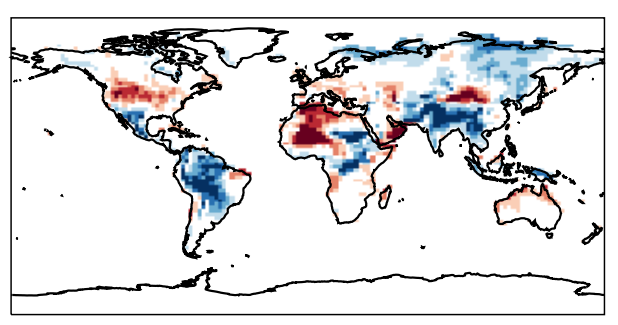

APR Diff. in QCAN ( -0.13)

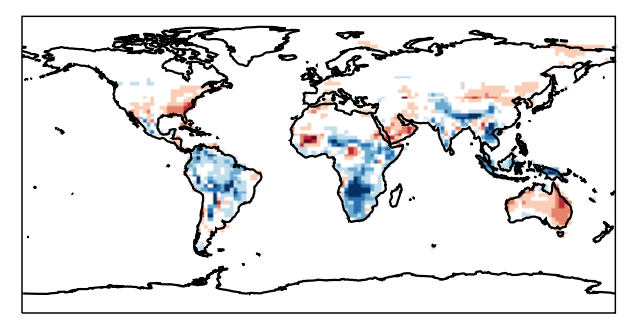

OCT Diff. in QCAN ( -0.25)

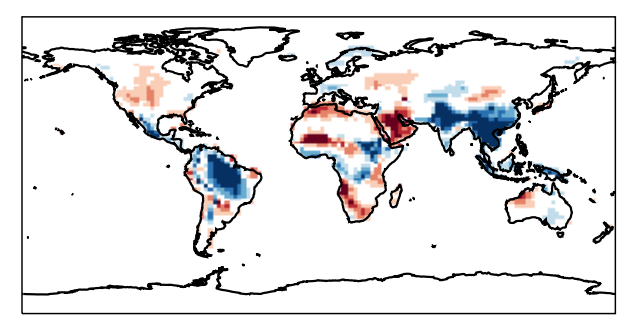

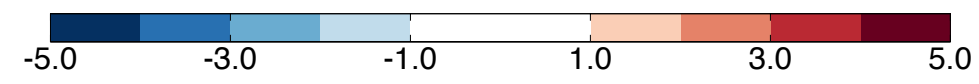

Figure S11. Differences of canopy humidity between ModelE2-YIBs and WFDEI for period 1996-2005. Each panel represents results averaged for a specific month. Units: $g$ $\mathrm{kg}^{-1}$. 
JAN Diff. in SOILM5 ( 0.04)

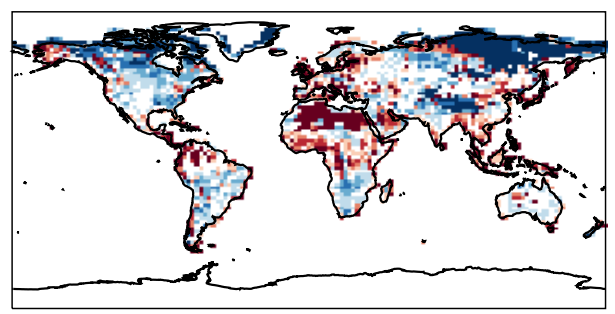

JUL Diff. in SOILM5 ( 0.07)

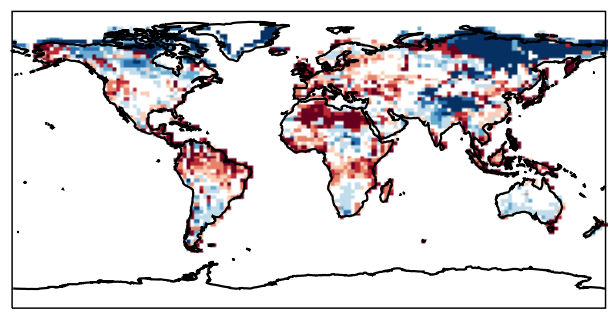

APR Diff. in SOILM5 ( 0.06)

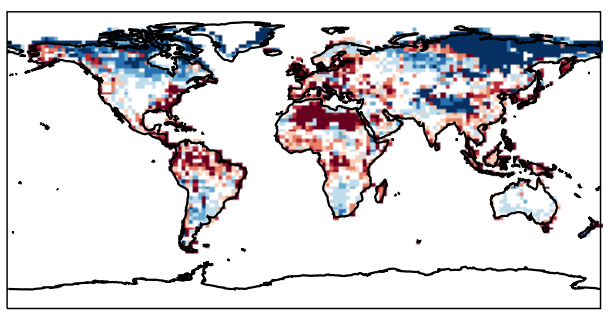

OCT Diff. in SOILM5 ( 0.07)

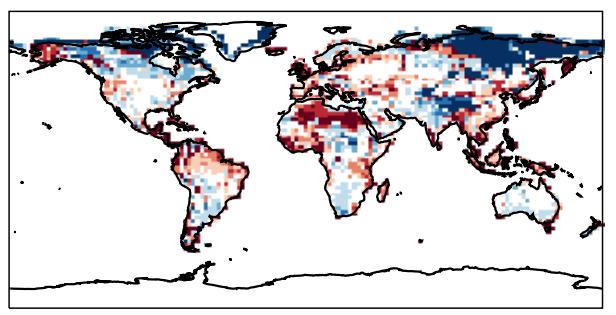

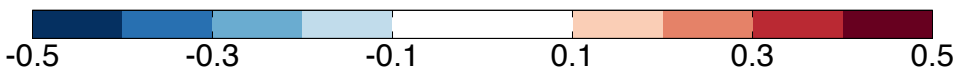

Figure S12. Differences of soil wetness at the $5^{\text {th }}$ soil layer $(\sim 1.5 \mathrm{~m})$ between ModelE2YIBs and WFDEI for period 1996-2005. Each panel represents results averaged for a specific month. Units: fraction. 
Table S1. Descriptions of 145 flux tower sites from NACP and FLUXNET a

\begin{tabular}{|c|c|c|c|c|c|c|c|}
\hline Site & PFT & Start & End & Longitude & Latitude & Country & Source \\
\hline AT-Neu & GRA & 2002 & 2004 & $11.32^{\circ} \mathrm{E}$ & $47.12^{\circ} \mathrm{N}$ & Austria & FLUXNET \\
\hline AU-Fog & SHR & 2006 & 2007 & $131.31^{\circ} \mathrm{E}$ & $12.54^{\circ} \mathrm{S}$ & Australia & FLUXNET \\
\hline AU-How & SHR & 2001 & 2006 & $131.15^{\circ} \mathrm{E}$ & $12.49^{\circ} \mathrm{S}$ & Australia & FLUXNET \\
\hline AU-Tum & $\mathrm{EBF}$ & 2001 & 2006 & $148.15^{\circ} \mathrm{E}$ & $35.66^{\circ} \mathrm{S}$ & Australia & FLUXNET \\
\hline AU-Wac & $\mathrm{EBF}$ & 2005 & 2007 & $145.19^{\circ} \mathrm{E}$ & $37.43^{\circ} \mathrm{S}$ & Australia & FLUXNET \\
\hline BE-Bra & ENF & 1997 & 2006 & $4.52^{\circ} \mathrm{E}$ & $51.31^{\circ} \mathrm{N}$ & Belgium & FLUXNET \\
\hline BE-Jal & ENF & 2006 & 2006 & $6.07^{\circ} \mathrm{E}$ & $50.56^{\circ} \mathrm{N}$ & Belgium & FLUXNET \\
\hline BE-Lon & $\mathrm{CRO}$ & 2004 & 2006 & $4.75^{\circ} \mathrm{E}$ & $50.55^{\circ} \mathrm{N}$ & Belgium & FLUXNET \\
\hline BE-Vie & ENF & 1996 & 2006 & $6^{\circ} \mathrm{E}$ & $50.31^{\circ} \mathrm{N}$ & Belgium & FLUXNET \\
\hline BR-Sa3 & $\mathrm{EBF}$ & 2000 & 2003 & $54.97^{\circ} \mathrm{W}$ & $3.02^{\circ} \mathrm{S}$ & Brazil & FLUXNET \\
\hline BW-Ma1 & SHR & 1999 & 2001 & $23.56^{\circ} \mathrm{E}$ & $19.92^{\circ} \mathrm{S}$ & Botswana & FLUXNET \\
\hline CA-Ca1 & ENF & 1998 & 2006 & $125.33^{\circ} \mathrm{W}$ & $49.87^{\circ} \mathrm{N}$ & Canada & NACP \\
\hline $\mathrm{CA}-\mathrm{Ca} 2$ & ENF & 2001 & 2006 & $125.29^{\circ} \mathrm{W}$ & $49.87^{\circ} \mathrm{N}$ & Canada & NACP \\
\hline $\mathrm{CA}-\mathrm{Ca} 3$ & ENF & 2002 & 2006 & $124.9^{\circ} \mathrm{W}$ & $49.54^{\circ} \mathrm{N}$ & Canada & NACP \\
\hline CA-Gro & ENF & 2004 & 2006 & $82.16^{\circ} \mathrm{W}$ & $48.22^{\circ} \mathrm{N}$ & Canada & NACP \\
\hline CA-Let & GRA & 1997 & 2007 & $112.94^{\circ} \mathrm{W}$ & $49.71^{\circ} \mathrm{N}$ & Canada & NACP \\
\hline CA-Man & ENF & 1994 & 2003 & $98.48^{\circ} \mathrm{W}$ & $55.88^{\circ} \mathrm{N}$ & Canada & FLUXNET \\
\hline CA-Mer & SHR & 1999 & 2006 & $75.52^{\circ} \mathrm{W}$ & $45.41^{\circ} \mathrm{N}$ & Canada & NACP \\
\hline CA-NS1 & ENF & 2002 & 2005 & $98.48^{\circ} \mathrm{W}$ & $55.88^{\circ} \mathrm{N}$ & Canada & FLUXNET \\
\hline CA-NS2 & ENF & 2001 & 2005 & $98.53^{\circ} \mathrm{W}$ & $55.91^{\circ} \mathrm{N}$ & Canada & FLUXNET \\
\hline CA-NS3 & ENF & 2001 & 2005 & $98.38^{\circ} \mathrm{W}$ & $55.91^{\circ} \mathrm{N}$ & Canada & FLUXNET \\
\hline CA-NS4 & ENF & 2002 & 2004 & $98.38^{\circ} \mathrm{W}$ & $55.91^{\circ} \mathrm{N}$ & Canada & FLUXNET \\
\hline CA-NS5 & ENF & 2001 & 2005 & $98.49^{\circ} \mathrm{W}$ & $55.86^{\circ} \mathrm{N}$ & Canada & FLUXNET \\
\hline CA-NS6 & SHR & 2001 & 2005 & $98.96^{\circ} \mathrm{W}$ & $55.92^{\circ} \mathrm{N}$ & Canada & FLUXNET \\
\hline CA-NS7 & SHR & 2002 & 2005 & $99.95^{\circ} \mathrm{W}$ & $56.64^{\circ} \mathrm{N}$ & Canada & FLUXNET \\
\hline CA-Oas & $\mathrm{DBF}$ & 1997 & 2006 & $106.2^{\circ} \mathrm{W}$ & $53.63^{\circ} \mathrm{N}$ & Canada & NACP \\
\hline CA-Obs & ENF & 2000 & 2006 & $105.12^{\circ} \mathrm{W}$ & $53.99^{\circ} \mathrm{N}$ & Canada & NACP \\
\hline CA-Ojp & ENF & 2000 & 2006 & $104.69^{\circ} \mathrm{W}$ & $53.92^{\circ} \mathrm{N}$ & Canada & NACP \\
\hline CA-Qcu & ENF & 2001 & 2006 & $74.04^{\circ} \mathrm{W}$ & $49.27^{\circ} \mathrm{N}$ & Canada & FLUXNET \\
\hline CA-Qfo & ENF & 2004 & 2006 & $74.34^{\circ} \mathrm{W}$ & $49.69^{\circ} \mathrm{N}$ & Canada & NACP \\
\hline CA-SJ1 & ENF & 2002 & 2005 & $104.66^{\circ} \mathrm{W}$ & $53.91^{\circ} \mathrm{N}$ & Canada & NACP \\
\hline $\mathrm{CA}-\mathrm{SJ} 2$ & ENF & 2003 & 2006 & $104.65^{\circ} \mathrm{W}$ & $53.95^{\circ} \mathrm{N}$ & Canada & NACP \\
\hline CA-SJ3 & ENF & 2005 & 2006 & $104.65^{\circ} \mathrm{W}$ & $53.88^{\circ} \mathrm{N}$ & Canada & NACP \\
\hline CA-TP4 & ENF & 2002 & 2007 & $80.36^{\circ} \mathrm{W}$ & $42.71^{\circ} \mathrm{N}$ & Canada & NACP \\
\hline CA-WP1 & SHR & 2003 & 2007 & $112.47^{\circ} \mathrm{W}$ & $54.95^{\circ} \mathrm{N}$ & Canada & NACP \\
\hline CH-Oe1 & GRA & 2002 & 2006 & $7.73^{\circ} \mathrm{E}$ & $47.29^{\circ} \mathrm{N}$ & Switzerland & FLUXNET \\
\hline $\mathrm{CH}-\mathrm{Oe} 2$ & $\mathrm{CRO}$ & 2005 & 2005 & $7.73^{\circ} \mathrm{E}$ & $47.29^{\circ} \mathrm{N}$ & Switzerland & FLUXNET \\
\hline CZ-BK1 & ENF & 2000 & 2006 & $18.54^{\circ} \mathrm{E}$ & $49.5^{\circ} \mathrm{N}$ & Czech Republic & FLUXNET \\
\hline DE-Bay & ENF & 1996 & 1999 & $11.87^{\circ} \mathrm{E}$ & $50.14^{\circ} \mathrm{N}$ & Germany & FLUXNET \\
\hline
\end{tabular}


Table S1. Continued.

\begin{tabular}{|c|c|c|c|c|c|c|c|}
\hline Site & PFT & Start & End & Longitude & Latitude & Country & Source \\
\hline DE-Geb & $\mathrm{CRO}$ & 2004 & 2006 & $10.91^{\circ} \mathrm{E}$ & $51.1^{\circ} \mathrm{N}$ & Germany & FLUXNET \\
\hline DE-Gri & GRA & 2005 & 2006 & $13.51^{\circ} \mathrm{E}$ & $50.95^{\circ} \mathrm{N}$ & Germany & FLUXNET \\
\hline DE-Hai & $\mathrm{DBF}$ & 2000 & 2006 & $10.45^{\circ} \mathrm{E}$ & $51.08^{\circ} \mathrm{N}$ & Germany & FLUXNET \\
\hline DE-Kli & $\mathrm{CRO}$ & 2004 & 2006 & $13.52^{\circ} \mathrm{E}$ & $50.89^{\circ} \mathrm{N}$ & Germany & FLUXNET \\
\hline DE-Meh & GRA & 2003 & 2006 & $10.66^{\circ} \mathrm{E}$ & $51.28^{\circ} \mathrm{N}$ & Germany & FLUXNET \\
\hline DE-Tha & ENF & 1996 & 2006 & $13.57^{\circ} \mathrm{E}$ & $50.96^{\circ} \mathrm{N}$ & Germany & FLUXNET \\
\hline DE-Wet & ENF & 2002 & 2006 & $11.46^{\circ} \mathrm{E}$ & $50.45^{\circ} \mathrm{N}$ & Germany & FLUXNET \\
\hline DK-Lva & GRA & 2005 & 2006 & $12.08^{\circ} \mathrm{E}$ & $55.68^{\circ} \mathrm{N}$ & Denmark & FLUXNET \\
\hline DK-Ris & $\mathrm{CRO}$ & 2004 & 2005 & $12.1^{\circ} \mathrm{E}$ & $55.53^{\circ} \mathrm{N}$ & Denmark & FLUXNET \\
\hline DK-Sor & $\mathrm{DBF}$ & 1996 & 2006 & $11.65^{\circ} \mathrm{E}$ & $55.49^{\circ} \mathrm{N}$ & Denmark & FLUXNET \\
\hline ES-ES1 & ENF & 1999 & 2006 & $0.32^{\circ} \mathrm{W}$ & $39.35^{\circ} \mathrm{N}$ & Spain & FLUXNET \\
\hline ES-ES2 & $\mathrm{CRO}$ & 2004 & 2006 & $0.32^{\circ} \mathrm{W}$ & $39.28^{\circ} \mathrm{N}$ & Spain & FLUXNET \\
\hline ES-LMa & SHR & 2004 & 2006 & $5.77^{\circ} \mathrm{W}$ & $39.94^{\circ} \mathrm{N}$ & Spain & FLUXNET \\
\hline ES-VDA & GRA & 2004 & 2006 & $1.45^{\circ} \mathrm{E}$ & $42.15^{\circ} \mathrm{N}$ & Spain & FLUXNET \\
\hline FI-Hyy & ENF & 1996 & 2006 & $24.3^{\circ} \mathrm{E}$ & $61.85^{\circ} \mathrm{N}$ & Finland & FLUXNET \\
\hline FI-Kaa & SHR & 2000 & 2006 & $27.3^{\circ} \mathrm{E}$ & $69.14^{\circ} \mathrm{N}$ & Finland & FLUXNET \\
\hline FI-Sod & ENF & 2000 & 2006 & $26.64^{\circ} \mathrm{E}$ & $67.36^{\circ} \mathrm{N}$ & Finland & FLUXNET \\
\hline FR-Fon & $\mathrm{DBF}$ & 2005 & 2006 & $2.78^{\circ} \mathrm{E}$ & $48.48^{\circ} \mathrm{N}$ & France & FLUXNET \\
\hline FR-Gri & $\mathrm{CRO}$ & 2005 & 2006 & $1.95^{\circ} \mathrm{E}$ & $48.84^{\circ} \mathrm{N}$ & France & FLUXNET \\
\hline FR-Hes & $\mathrm{DBF}$ & 1997 & 2006 & $7.07^{\circ} \mathrm{E}$ & $48.67^{\circ} \mathrm{N}$ & France & FLUXNET \\
\hline FR-LBr & ENF & 1996 & 2006 & $0.77^{\circ} \mathrm{W}$ & $44.72^{\circ} \mathrm{N}$ & France & FLUXNET \\
\hline FR-Lq1 & GRA & 2004 & 2006 & $2.74^{\circ} \mathrm{E}$ & $45.64^{\circ} \mathrm{N}$ & France & FLUXNET \\
\hline FR-Lq2 & GRA & 2004 & 2006 & $2.74^{\circ} \mathrm{E}$ & $45.64^{\circ} \mathrm{N}$ & France & FLUXNET \\
\hline FR-Pue & $\mathrm{EBF}$ & 2000 & 2006 & $3.6^{\circ} \mathrm{E}$ & $43.74^{\circ} \mathrm{N}$ & France & FLUXNET \\
\hline HU-Bug & GRA & 2002 & 2006 & $19.6^{\circ} \mathrm{E}$ & $46.69^{\circ} \mathrm{N}$ & Hungary & FLUXNET \\
\hline HU-Mat & GRA & 2004 & 2006 & $19.73^{\circ} \mathrm{E}$ & $47.85^{\circ} \mathrm{N}$ & Hungary & FLUXNET \\
\hline ID-Pag & $\mathrm{EBF}$ & 2002 & 2003 & $114.04^{\circ} \mathrm{E}$ & $2.35^{\circ} \mathrm{N}$ & Indonesia & FLUXNET \\
\hline IE-Ca1 & CRO & 2004 & 2006 & $6.92^{\circ} \mathrm{W}$ & $52.86^{\circ} \mathrm{N}$ & Ireland & FLUXNET \\
\hline IE-Dri & GRA & 2003 & 2005 & $8.75^{\circ} \mathrm{W}$ & $51.99^{\circ} \mathrm{N}$ & Ireland & FLUXNET \\
\hline IL-Yat & ENF & 2001 & 2006 & $35.05^{\circ} \mathrm{E}$ & $31.35^{\circ} \mathrm{N}$ & Israel & FLUXNET \\
\hline IT-Amp & GRA & 2002 & 2006 & $13.61^{\circ} \mathrm{E}$ & $41.9^{\circ} \mathrm{N}$ & Italy & FLUXNET \\
\hline IT-BCi & CRO & 2004 & 2006 & $14.96^{\circ} \mathrm{E}$ & $40.52^{\circ} \mathrm{N}$ & Italy & FLUXNET \\
\hline IT-Col & $\mathrm{DBF}$ & 1996 & 2006 & $13.59^{\circ} \mathrm{E}$ & $41.85^{\circ} \mathrm{N}$ & Italy & FLUXNET \\
\hline IT-Cpz & $\mathrm{EBF}$ & 1997 & 2006 & $12.38^{\circ} \mathrm{E}$ & $41.71^{\circ} \mathrm{N}$ & Italy & FLUXNET \\
\hline IT-LMa & GRA & 2003 & 2006 & $7.16^{\circ} \mathrm{E}$ & $45.58^{\circ} \mathrm{N}$ & Italy & FLUXNET \\
\hline IT-Lav & ENF & 2000 & 2006 & $11.28^{\circ} \mathrm{E}$ & $45.96^{\circ} \mathrm{N}$ & Italy & FLUXNET \\
\hline IT-Lec & $\mathrm{EBF}$ & 2005 & 2006 & $11.27^{\circ} \mathrm{E}$ & $43.31^{\circ} \mathrm{N}$ & Italy & FLUXNET \\
\hline IT-MBo & GRA & 2003 & 2006 & $11.05^{\circ} \mathrm{E}$ & $46.02^{\circ} \mathrm{N}$ & Italy & FLUXNET \\
\hline IT-Mal & GRA & 2003 & 2006 & $11.7^{\circ} \mathrm{E}$ & $46.12^{\circ} \mathrm{N}$ & Italy & FLUXNET \\
\hline
\end{tabular}


Table S1. Continued.

\begin{tabular}{|c|c|c|c|c|c|c|c|}
\hline Site & PFT & Start & End & Longitude & Latitude & Country & Source \\
\hline IT-Non & $\mathrm{DBF}$ & 2001 & 2006 & $11.09^{\circ} \mathrm{E}$ & $44.69^{\circ} \mathrm{N}$ & Italy & FLUXNET \\
\hline IT-PT1 & $\mathrm{DBF}$ & 2002 & 2004 & $9.06^{\circ} \mathrm{E}$ & $45.2^{\circ} \mathrm{N}$ & Italy & FLUXNET \\
\hline IT-Pia & SHR & 2002 & 2005 & $10.08^{\circ} \mathrm{E}$ & $42.58^{\circ} \mathrm{N}$ & Italy & FLUXNET \\
\hline IT-Ren & ENF & 1999 & 2006 & $11.44^{\circ} \mathrm{E}$ & $46.59^{\circ} \mathrm{N}$ & Italy & FLUXNET \\
\hline IT-Ro1 & $\mathrm{DBF}$ & 2000 & 2006 & $11.93^{\circ} \mathrm{E}$ & $42.41^{\circ} \mathrm{N}$ & Italy & FLUXNET \\
\hline IT-Ro2 & $\mathrm{DBF}$ & 2002 & 2006 & $11.92^{\circ} \mathrm{E}$ & $42.39^{\circ} \mathrm{N}$ & Italy & FLUXNET \\
\hline IT-SRo & ENF & 1999 & 2006 & $10.28^{\circ} \mathrm{E}$ & $43.73^{\circ} \mathrm{N}$ & Italy & FLUXNET \\
\hline NL-Ca1 & GRA & 2003 & 2006 & $4.93^{\circ} \mathrm{E}$ & $51.97^{\circ} \mathrm{N}$ & Netherlands & FLUXNET \\
\hline NL-Hor & GRA & 2004 & 2006 & $5.07^{\circ} \mathrm{E}$ & $52.03^{\circ} \mathrm{N}$ & Netherlands & FLUXNET \\
\hline NL-Lan & CRO & 2005 & 2006 & $4.9^{\circ} \mathrm{E}$ & $51.95^{\circ} \mathrm{N}$ & Netherlands & FLUXNET \\
\hline NL-Loo & ENF & 1996 & 2006 & $5.74^{\circ} \mathrm{E}$ & $52.17^{\circ} \mathrm{N}$ & Netherlands & FLUXNET \\
\hline PL-wet & SHR & 2004 & 2005 & $16.31^{\circ} \mathrm{E}$ & $52.76^{\circ} \mathrm{N}$ & Poland & FLUXNET \\
\hline PT-Esp & $\mathrm{EBF}$ & 2002 & 2006 & $8.6^{\circ} \mathrm{W}$ & $38.64^{\circ} \mathrm{N}$ & Portugal & FLUXNET \\
\hline PT-Mi1 & $\mathrm{EBF}$ & 2003 & 2005 & $8^{\circ} \mathrm{W}$ & $38.54^{\circ} \mathrm{N}$ & Portugal & FLUXNET \\
\hline PT-Mi2 & GRA & 2004 & 2006 & $8.03^{\circ} \mathrm{W}$ & $38.48^{\circ} \mathrm{N}$ & Portugal & FLUXNET \\
\hline RU-Fyo & ENF & 1998 & 2004 & $32.92^{\circ} \mathrm{E}$ & $56.46^{\circ} \mathrm{N}$ & Russia & FLUXNET \\
\hline SE-Deg & SHR & 2001 & 2005 & $19.55^{\circ} \mathrm{E}$ & $64.18^{\circ} \mathrm{N}$ & Sweden & FLUXNET \\
\hline SE-Faj & SHR & 2005 & 2006 & $13.55^{\circ} \mathrm{E}$ & $56.27^{\circ} \mathrm{N}$ & Sweden & FLUXNET \\
\hline SE-Fla & ENF & 1996 & 2002 & $19.46^{\circ} \mathrm{E}$ & $64.11^{\circ} \mathrm{N}$ & Sweden & FLUXNET \\
\hline SE-Nor & ENF & 1996 & 2005 & $17.48^{\circ} \mathrm{E}$ & $60.09^{\circ} \mathrm{N}$ & Sweden & FLUXNET \\
\hline SE-Sk1 & ENF & 2005 & 2005 & $17.92^{\circ} \mathrm{E}$ & $60.13^{\circ} \mathrm{N}$ & Sweden & FLUXNET \\
\hline SE-Sk2 & ENF & 2004 & 2005 & $17.84^{\circ} \mathrm{E}$ & $60.13^{\circ} \mathrm{N}$ & Sweden & FLUXNET \\
\hline UK-AMo & SHR & 2005 & 2005 & $3.24^{\circ} \mathrm{W}$ & $55.79^{\circ} \mathrm{N}$ & UK & FLUXNET \\
\hline UK-EBu & GRA & 2004 & 2006 & $3.21^{\circ} \mathrm{W}$ & $55.87^{\circ} \mathrm{N}$ & UK & FLUXNET \\
\hline UK-ESa & CRO & 2003 & 2005 & $2.86^{\circ} \mathrm{W}$ & $55.91^{\circ} \mathrm{N}$ & UK & FLUXNET \\
\hline UK-Gri & ENF & 1997 & 2006 & $3.8^{\circ} \mathrm{W}$ & $56.61^{\circ} \mathrm{N}$ & UK & FLUXNET \\
\hline UK-Ham & $\mathrm{DBF}$ & 2004 & 2005 & $0.86^{\circ} \mathrm{W}$ & $51.15^{\circ} \mathrm{N}$ & UK & FLUXNET \\
\hline UK-PL3 & $\mathrm{DBF}$ & 2005 & 2006 & $1.27^{\circ} \mathrm{W}$ & $51.45^{\circ} \mathrm{N}$ & UK & FLUXNET \\
\hline US-ARM & GRA & 2000 & 2007 & $97.49^{\circ} \mathrm{W}$ & $36.6^{\circ} \mathrm{N}$ & US & NACP \\
\hline US-Aud & GRA & 2002 & 2006 & $110.51^{\circ} \mathrm{W}$ & $31.59^{\circ} \mathrm{N}$ & US & FLUXNET \\
\hline US-Bar & $\mathrm{DBF}$ & 2004 & 2005 & $71.29^{\circ} \mathrm{W}$ & $44.07^{\circ} \mathrm{N}$ & US & FLUXNET \\
\hline US-Bkg & GRA & 2004 & 2006 & $96.84^{\circ} \mathrm{W}$ & $44.35^{\circ} \mathrm{N}$ & US & FLUXNET \\
\hline US-Blo & ENF & 1997 & 2006 & $120.63^{\circ} \mathrm{W}$ & $38.9^{\circ} \mathrm{N}$ & US & FLUXNET \\
\hline US-Bo1 & CRO & 1996 & 2007 & $88.29^{\circ} \mathrm{W}$ & $40.01^{\circ} \mathrm{N}$ & US & FLUXNET \\
\hline US-Dk3 & ENF & 1998 & 2005 & $79.09^{\circ} \mathrm{W}$ & $35.98^{\circ} \mathrm{N}$ & US & NACP \\
\hline US-FPe & GRA & 2000 & 2006 & $105.1^{\circ} \mathrm{W}$ & $48.31^{\circ} \mathrm{N}$ & US & FLUXNET \\
\hline US-Goo & GRA & 2002 & 2006 & $89.87^{\circ} \mathrm{W}$ & $34.26^{\circ} \mathrm{N}$ & US & FLUXNET \\
\hline US-Ha1 & DBF & 1991 & 2006 & $72.17^{\circ} \mathrm{W}$ & $42.54^{\circ} \mathrm{N}$ & US & NACP \\
\hline US-Ho1 & ENF & 1996 & 2004 & $68.74^{\circ} \mathrm{W}$ & $45.2^{\circ} \mathrm{N}$ & US & NACP \\
\hline
\end{tabular}


Table S1. Continued.

\begin{tabular}{|c|c|c|c|c|c|c|c|}
\hline Site & PFT & Start & End & Longitude & Latitude & Country & Source \\
\hline US-Ho2 & ENF & 1999 & 2004 & $68.75^{\circ} \mathrm{W}$ & $45.21^{\circ} \mathrm{N}$ & US & FLUXNET \\
\hline US-IB1 & $\mathrm{CRO}$ & 2005 & 2007 & $88.22^{\circ} \mathrm{W}$ & $41.86^{\circ} \mathrm{N}$ & US & NACP \\
\hline US-IB2 & GRA & 2004 & 2007 & $88.24^{\circ} \mathrm{W}$ & $41.84^{\circ} \mathrm{N}$ & US & NACP \\
\hline US-Los & SHR & 2000 & 2006 & $89.98^{\circ} \mathrm{W}$ & $46.08^{\circ} \mathrm{N}$ & US & NACP \\
\hline US-MMS & DBF & 1999 & 2006 & $86.41^{\circ} \mathrm{W}$ & $39.32^{\circ} \mathrm{N}$ & US & NACP \\
\hline US-MOz & DBF & 2004 & 2007 & $92.2^{\circ} \mathrm{W}$ & $38.74^{\circ} \mathrm{N}$ & US & NACP \\
\hline US-Me2 & ENF & 2002 & 2007 & $121.56^{\circ} \mathrm{W}$ & $44.45^{\circ} \mathrm{N}$ & US & NACP \\
\hline US-Me3 & ENF & 2004 & 2005 & $121.61^{\circ} \mathrm{W}$ & $44.32^{\circ} \mathrm{N}$ & US & NACP \\
\hline US-Me4 & ENF & 1996 & 2000 & $121.62^{\circ} \mathrm{W}$ & $44.5^{\circ} \mathrm{N}$ & US & FLUXNET \\
\hline US-Me5 & ENF & 1999 & 2002 & $121.57^{\circ} \mathrm{W}$ & $44.44^{\circ} \mathrm{N}$ & US & NACP \\
\hline US-NR1 & ENF & 1998 & 2007 & $105.55^{\circ} \mathrm{W}$ & $40.03^{\circ} \mathrm{N}$ & US & NACP \\
\hline US-Ne1 & $\mathrm{CRO}$ & 2001 & 2006 & $96.48^{\circ} \mathrm{W}$ & $41.17^{\circ} \mathrm{N}$ & US & NACP \\
\hline US-Ne2 & $\mathrm{CRO}$ & 2001 & 2006 & $96.47^{\circ} \mathrm{W}$ & $41.17^{\circ} \mathrm{N}$ & US & NACP \\
\hline US-Ne3 & $\mathrm{CRO}$ & 2001 & 2006 & $96.44^{\circ} \mathrm{W}$ & $41.18^{\circ} \mathrm{N}$ & US & NACP \\
\hline US-Oho & DBF & 2004 & 2005 & $83.84^{\circ} \mathrm{W}$ & $41.56^{\circ} \mathrm{N}$ & US & FLUXNET \\
\hline US-PFa & ENF & 1995 & 2005 & $90.27^{\circ} \mathrm{W}$ & $45.95^{\circ} \mathrm{N}$ & US & NACP \\
\hline US-SO2 & SHR & 1998 & 2006 & $116.62^{\circ} \mathrm{W}$ & $33.37^{\circ} \mathrm{N}$ & US & NACP \\
\hline US-SP1 & ENF & 2000 & 2005 & $82.22^{\circ} \mathrm{W}$ & $29.74^{\circ} \mathrm{N}$ & US & FLUXNET \\
\hline US-SP2 & ENF & 1998 & 2004 & $82.25^{\circ} \mathrm{W}$ & $29.77^{\circ} \mathrm{N}$ & US & FLUXNET \\
\hline US-SP3 & ENF & 1999 & 2004 & $82.16^{\circ} \mathrm{W}$ & $29.76^{\circ} \mathrm{N}$ & US & FLUXNET \\
\hline US-Shd & GRA & 1997 & 2000 & $96.68^{\circ} \mathrm{W}$ & $36.93^{\circ} \mathrm{N}$ & US & NACP \\
\hline US-Syv & ENF & 2001 & 2006 & $89.35^{\circ} \mathrm{W}$ & $46.24^{\circ} \mathrm{N}$ & US & NACP \\
\hline US-Ton & SHR & 2001 & 2007 & $120.97^{\circ} \mathrm{W}$ & $38.43^{\circ} \mathrm{N}$ & US & NACP \\
\hline US-UMB & DBF & 1999 & 2006 & $84.71^{\circ} \mathrm{W}$ & $45.56^{\circ} \mathrm{N}$ & US & NACP \\
\hline US-Var & GRA & 2000 & 2007 & $120.95^{\circ} \mathrm{W}$ & $38.41^{\circ} \mathrm{N}$ & US & NACP \\
\hline US-WBW & DBF & 1995 & 1999 & $84.29^{\circ} \mathrm{W}$ & $35.96^{\circ} \mathrm{N}$ & US & FLUXNET \\
\hline US-WCr & DBF & 1998 & 2006 & $90.08^{\circ} \mathrm{W}$ & $45.81^{\circ} \mathrm{N}$ & US & NACP \\
\hline ZA-Kru & SHR & 2001 & 2003 & $31.5^{\circ} \mathrm{E}$ & $25.02^{\circ} \mathrm{S}$ & South Africa & FLUXNET \\
\hline
\end{tabular}

${ }^{a}$ The NACP site information is adopted from Schaefer et al. (2012), except that the operational time span listed here is only for the period when measurements of GPP are available. Most of NACP sites are also part of the FLUXNET network.

${ }^{\mathrm{b}}$ PFT names are: evergreen needleleaf forest (ENF), deciduous broadleaf forest (DBF), shrublands (SHR), grasslands (GRA), and croplands (CRO). Some site biome definitions are attributed to the closet model PFT. For example, closed shrublands, permanent wetlands, and woody savannas are attributed to shrublands. Mixed forests are attributed to ENF. 
Table S2. Statistics of site-level GPP $\left(\mathrm{g}[\mathrm{C}]\right.$ day $\left.^{-1}\right)$ and NEE $\left(\mathrm{g}[\mathrm{C}]\right.$ day $\left.^{-1}\right)$ for different PFTs

\begin{tabular}{ccccccc}
\hline PFT & ENF & EBF & DBF & SHR & GRA & CRO \\
\hline GPP Observed & $4.0 \pm 2.1^{\text {a }}$ & $7.4 \pm 3.1$ & $5.1 \pm 1.6$ & $2.8 \pm 1.5$ & $4.4 \pm 1.8$ & $5.1 \pm 1.3$ \\
GPP Modeled & $3.9 \pm 1.2$ & $6.3 \pm 3.1$ & $4.4 \pm 0.9$ & $2.8 \pm 1.7$ & $3.5 \pm 1.4$ & $3.6 \pm 1.1$ \\
NEE Observed & $-0.4 \pm 0.9$ & $-0.7 \pm 1.1$ & $-1.2 \pm 0.7$ & $-0.2 \pm 0.5$ & $-0.5 \pm 0.4$ & $-0.8 \pm 0.4$ \\
GPP Modeled & $-0.0 \pm 0.4$ & $-0.0 \pm 0.4$ & $-0.1 \pm 0.2$ & $-0.3 \pm 0.5$ & $-0.4 \pm 0.3$ & $-0.1 \pm 0.3$ \\
\hline
\end{tabular}

${ }^{a}$ The numbers are denoted as $\mathrm{A} \pm \sigma$, where $\mathrm{A}$ is the mean value at all sites with the same PFT and $\sigma$ is corresponding standard deviation. 


\section{References}

Batjes, N. H.: Harmonized soil profile data for applications at global and continental scales: updates to the WISE database, Soil Use Manage, 25, 124-127, doi:10.1111/J.1475-2743.2009.00202.X, 2009.

Huntzinger, D. N., Schwalm, C., Michalak, A. M., Schaefer, K., King, A. W., Wei, Y., Jacobson, A., Liu, S., Cook, R. B., Post, W. M., Berthier, G., Hayes, D., Huang, M., Ito, A., Lei, H., Lu, C., Mao, J., Peng, C. H., Peng, S., Poulter, B., Riccuito, D., Shi, X., Tian, H., Wang, W., Zeng, N., Zhao, F., and Zhu, Q.: The North American Carbon Program Multi-Scale Synthesis and Terrestrial Model Intercomparison Project - Part 1: Overview and experimental design, Geosci Model Dev, 6, 2121 2133, doi:10.5194/Gmd-6-2121-2013, 2013.

Piao, S. L., Sitch, S., Ciais, P., Friedlingstein, P., Peylin, P., Wang, X. H., Ahlstrom, A., Anav, A., Canadell, J. G., Cong, N., Huntingford, C., Jung, M., Levis, S., Levy, P. E., Li, J. S., Lin, X., Lomas, M. R., Lu, M., Luo, Y. Q., Ma, Y. C., Myneni, R. B., Poulter, B., Sun, Z. Z., Wang, T., Viovy, N., Zaehle, S., and Zeng, N.: Evaluation of terrestrial carbon cycle models for their response to climate variability and to $\mathrm{CO} 2$ trends, Global Change Biol, 19, 2117-2132, doi:10.1111/Gcb.12187, 2013.

Schaefer, K. and co-authors: A model-data comparison of gross primary productivity: Results from the North American Carbon Program site synthesis, J. Geophys. Res., 117, G03010, doi:10.1029/2012jg001960, 2012.

Sitch, S., Friedlingstein, P., Gruber, N., Jones, S. D., Murray-Tortarolo, G., Ahlström, A., Doney, S. C., Graven, H., Heinze, C., Huntingford, C., Levis, S., Levy, P. E., Lomas, M., Poulter, B., Viovy, N., Zaehle, S., Zeng, N., Arneth, A., Bonan, G., Bopp, L., Canadell, J. G., Chevallier, F., Ciais, P., Ellis, R., Gloor, M., Peylin, P., Piao, S. L., Quéré, C. L., Smith, B., Zhu, Z., and Myneni, R.: Recent trends and drivers of regional sources and sinks of carbon dioxide, Biogeosciences, 12, 653-679, 2015.

Wutzler, T. and Reichstein, M.: Soils apart from equilibrium - consequences for soil carbon balance modelling, Biogeosciences, 4, 125-136, 2007. 